\title{
SPATIAL DISTRIBUTION OF LYMNAEIDAE (MOLLUSCA, BASOMMATOPHORA), INTERMEDIATE HOST OF Fasciola hepatica LINNAEUS, 1758 (TREMATODA, DIGENEA) IN BRAZIL
}

Camilla MEDEIROS(1,2,3), Ronaldo Guilherme Carvalho SCHOLTE(2), Sthefane D’ÁVILA(3,4), Roberta Lima CALDEIRA(1,2) \& Omar dos Santos CARVALHO(2)

\begin{abstract}
SUMMARY
Snails of the family Lymnaeidae act as intermediate hosts in the biological cycle of Fasciola hepatica, which is a biological agent of fasciolosis, a parasitic disease of medical importance for humans and animals. The present work aimed to update and map the spatial distribution of the intermediate host snails of $F$. hepatica in Brazil. Data on the distribution of lymnaeids species were compiled from the Collection of Medical Malacology (Fiocruz-CMM, CPqRR), Collection of Malacology (MZUSP), "SpeciesLink" (CRIA) network and through systematic surveys in the literature. Our maps of the distribution of lymnaeids show that Pseudosuccinea columella is the most common species and it is widespread in the South and Southeast with few records in the Midwest, North and Northeast regions. The distribution of the Galba viatrix, G. cubensis and G. truncatula showed a few records in the South and Southeast regions, they were not reported for the Midwest, North and Northeast. In addition, in the South region there are a few records for G. viatrix and one occurrence of Lymnaea rupestris. Our findings resulted in the first map of the spatial distribution of Lymnaeidae species in Brazil which might be useful to better understand the fasciolosis distribution and delineate priority areas for control interventions.
\end{abstract}

KEYWORDS: Lymnaeids; Fasciolosis; Spatial distribution; Snails; Brazil.

\section{INTRODUCTION}

Snails of the family Lymnaeidae are small Basommatophora hermaphrodites, approximately $10 \mathrm{~mm}$ long and $6 \mathrm{~mm}$ wide. The lymnaeids species is widely distributed around the tropical and subtropical areas of the Americas, Europe, Asia, Africa, and Oceania ${ }^{36}$ and inhabits natural (streams, ponds, swamps) or artificial (irrigation ditches, small dams, floodplain) types of water collections ${ }^{23}$.

The importance of the lymnaeids is due to the fact that they act as intermediate hosts in the biological cycle of the digenetic trematode Fasciola hepatica Linnaeus, 1758 (Trematoda, Digenea), biological agent of fasciolosis, which is a parasitic disease of medical importance for both humans and animals.

Based on an increasing number of human cases since 1980, MASCOMA et al. ${ }^{50}$ proposed that fasciolosis should be considered an important parasitic human disease rather than just a secondary zoonotic disease with an estimated number of infected people of more than 2.4 million people worldwide ${ }^{108}$. The economic losses caused by this disease include a significant impact on the livestock industry due to the high costs for therapeutic treatments in cattle, goat, and sheep breeding $24,29,43,49,83$. SMOOKER et al. ${ }^{84}$ calculated a global cost of more than two billion dollars a year because of the infection.
In Brazil, the lymnaeids species already recorded are: Pseudosuccinea columella (Say, 1817), Galba viatrix d'Orbigny, 1835, Galba truncatula (Müeller, 1774), Galba cubensis Pfeiffer, 1839 and Lymnaea rupestris Paraense, 1982. Except for $L$. rupestris, the other species have proved to be susceptible to infection by $F$. hepatica. Regarding the identification of lymnaeids species, their systematics is confused and there are a lot of difficulties in identifying some species using only morphological characters $^{28,77}$, so molecular markers are frequently necessary. Here, we used the taxonomy proposed by BAKER ${ }^{13}$ for the species $P$. columella.

The present work aimed to update and map the spatial distribution of the intermediate host snails of F. hepatica in Brazil.

\section{MATERIAL AND METHODS}

Malacological data: Data on the distribution of lymnaeids species were obtained from: i) Collection of Medical Malacology (FiocruzCMM) of the Laboratory of Medical Helminthology and Malacology, René Rachou Research Center (Fiocruz/MG); ii) Collection of Malacology of the Museum of Zoology of University of São Paulo (MZUSP); iii) "SpeciesLink" (CRIA) network (http://www.splink. org.br/), by using the keyword Lymnaeidae in the database search; iv) systematic surveys in scientific articles, gray literature, dissertations, theses, and communications, by using the combined keywords: Lymnaea/

(1) Programa de Pós-Graduação em Ciências da Saúde, Doenças Infecciosas e Parasitárias, Centro de Pesquisas René Rachou, Fiocruz, Belo Horizonte, Minas Gerais, Brazil.

(2) Laboratório de Helmintologia e Malacologia Médica, Centro de Pesquisas René Rachou, Fiocruz, Belo Horizonte, Minas Gerais, Brazil.

(3) Museu de Malacologia Prof. Maury Pinto de Oliveira, Instituto de Ciências Biológicas, Universidade Federal de Juiz de Fora, Juiz de Fora, Minas Gerais, Brazil.

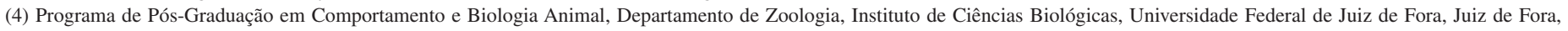
Minas Gerais, Brazil.

Correspondence to: Camilla Medeiros, Tel: +55-3133497816. E-mail: ccarvalho@cpqrr.fiocruz.br 


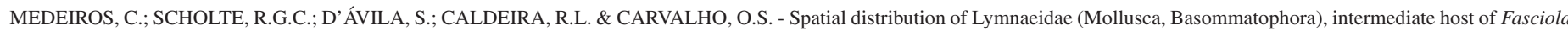
hepatica Linnaeus, 1758 (Trematoda, Digenea) in Brazil. Rev. Inst. Med. Trop. Sao Paulo, 56(3): 235-52, 2014.

Galba/Pseudosuccinea/Lymnaeidae + occurrence + Brazil; Lymnaeal Galba/Pseudosuccinea/Lymnaeidae + distribution + Brazil; Lymnaea/ Galba/Pseudosuccinea/Lymnaeidae + record + Brazil; Lymnaea/Galba/ Pseudosuccinea/Lymnaeidae + collection + Brazil; Fasciola + occurrence + Brazil; Fasciola + distribution + Brazil; Fasciola + record + Brazil; Fasciolosis + occurrence + Brazil; Fasciolosis + distribution + Brazil and Fasciolosis + record + Brazil. According to the type of database (or sites), terms were used in English or Portuguese: Google scholar, Web of Knowledge, Scielo, PubMed, Periódicos Capes (Capes Journals) and Banco Digital de Teses e Dissertações (Theses and Dissertations Digital Database). The data collected had information relative to the occurrence of lymnaeids per state and municipalities of Brazilian regions and if the snails were found naturally infected by $F$. hepatica.

In the majority of the data used, the identification of the lymnaeids species was made using only morphological characters except when the data are from Fiocruz-CMM, where molecular markers were utilized to confirm the morphological identification. The molecular technique used was the polymerase chain reaction and restriction fragment length polymorphism (PCR-RFLP) targeted to the first and second internal transcribed spacers (ITS1 and ITS2) rDNA and to the mitochondrial 16S ribosomal gene $(16 \mathrm{~S} \mathrm{rDNAmt})^{19,22}$.

Data management and mapping: Data management, visualization and mapping were carried out in ArcGIS version 9.3 (ESRI; Redlands, CA, USA).

\section{RESULTS}

Reports on five species of Lymnaeidae were found in Brazil. Tables 1 and 2 summarize the distribution per locality of species in the five regions of Brazil (Fig. 1 and 2), South (Fig. 3), Southeast (Fig. 4), Midwest, North and Northeast regions (Fig. 5). In the "SpeciesLink" (CRIA) network, we found data relative to four collections: Collection of Molluscs of Museum of Science and Technology, PUCRS; Collection of Molluscs of National Institute of Research of Amazônia, INPA; Malacological Collection of Biological Science Department, DCBio/UFES and Collection of Malacology of Oswaldo Cruz Institute, Fiocruz-CMIOC. The information about the localities where the specimens occurred was not available from the Collection of Malacology of Oswaldo Cruz Institute, Fiocruz-CMIOC, because of this, we did not add these data to our results. The data from the Collection of Medical Malacology, Fiocruz-CMM, were already available to us.

Lymnaeids snails were reported in 417 municipalities and one ecological reserve (Taim) of 16 states, which corresponds to $7.5 \%$ of all municipalities in Brazil. Pseudosuccinea columella was reported in $400(95.7 \%)$ localities while G. viatrix in $10(2.4 \%)$, G. cubensis in six $(1.4 \%), G$. truncatula in three $(0.7 \%)$ and $L$. rupestris in one $(0.2 \%)$. In 25 (6\%) municipalities: Bom Jesus, Cambará do Sul, Erval Grande, Estrela, Ibirubá, Porto Alegre, São Borja, São Francisco de Paula, Selbach, Três Coroas, Vacaria and Viamão of Rio Grande do Sul State; Piracicaba, Presidente Prudente, São Paulo and Ubatuba of São Paulo State; Rio de Janeiro, Nova Friburgo, Teresópolis and Vassouras of Rio de Janeiro State; Ouro Branco and Careaçu of Minas Gerais State; Campos Novos/SC; Curitiba/PR and Belém/PA, it was not possible to accomplish a complete identification of the specimens collected. Occurrence of more than one species was reported in 13 municipalities (3.1\%): Dom Pedrito, Pelotas and Santa Vitória do Palmar in the state of Rio Grande do Sul; Florianópolis ( $P$. columella and G. viatrix) and Seara (L. rupestris, $P$. columella and $G$. viatrix) in the state of Santa Catarina; Teresópolis ( $P$. columella; G. cubensis and G. truncatula), Nova Friburgo (P. columella and G. truncatula), Paraíba do Sul, Petrópolis, Rio de Janeiro and Três Rios (P. columella and G. cubensis) in the state of Rio de Janeiro; Belo Horizonte (P. columella, G. viatrix and G. cubensis) and Rio Acima ( $P$. columella, $G$. viatrix and $G$. truncatula) in the state of Minas Gerais.

Specimens of $P$. columella and $G$. viatrix were reported in 403 localities $(96.4 \%)$. P. columella was reported naturally infected with $F$. hepatica in 11 municipalities of the states of Rio Grande do Sul, São Paulo, Minas Gerais and Rio de Janeiro, G. viatrix was found naturally infected in three municipalities of Rio Grande do Sul while Lymnaea $s p$. was found in one municipality of Minas Gerais. In Dom Pedrito/RS, both species were found naturally infected.

\section{DISCUSSION}

Our study resulted in the first map of the spatial distribution of Lymnaeidae species, intermediate host snails of $F$. hepatica in Brazil. The presence of host snails could reflect the disease distribution, and these data are essential for describing the risk areas of fasciolosis since there is an increase of the association between the global number of human cases and the presence of the intermediate host snail ${ }^{50}$.

PARAENSE ${ }^{65}$ showed that $G$. viatrix had few records in the South and $P$. columella was distributed mainly in the South and Southeast, where a high number of fasciolosis cases occurs ${ }^{36}$, with few records in the Midwest region. After that, the same author reported two new localities of $P$. columella for the states of Amazonas and Bahia ${ }^{67}$ in the North and Northeast respectively. This agrees with our study where we also reported a low presence of this species in these regions. Our final maps about spatial distribution of lymnaeids snails show that $P$. columella is the most common species and it is widespread in the South and Southeast with few records in the Midwest, North and Northeast regions. Galba viatrix is predominantly distributed in the South (eight records) with only two records for the Southeast region (Belo Horizonte and Rio Acima, Minas Gerais State). The distribution of G. viatrix, G. cubensis and G. truncatula comprises few records in Southeast and South regions. They were not reported for the Midwest, North and Northeast. For the South region, the species L. rupestris was only described and found in the Nova Teutônia district in Seara municipality at Santa Catarina State ${ }^{64}$.

Despite the few records showed by $G$. viatrix in our study, according to MATTOS \& $\mathrm{UENO}^{52}$ this intermediate host is considered the most efficient species in the transmission of $F$. hepatica due to its high susceptibility when compared with $P$. columella $a^{52}$. However, $P$. columella showed to be the most distributed species (Fig. 1; 95.7\%) and is certainly the most important intermediate host in Brazil. This is probably due to its biological aspects, such as its high capacity for self-fertilization, favorable condition in dispersion and colonization of new habitats ${ }^{20,35}$. Pseudosuccinea columella is commonly found in most human or animal fasciolosis outbreaks while the occurrence of $G$. viatrix is, currently, restricted to the state of Rio Grande do Sul $24,33,63,76,101$ and in Minas Gerais ${ }^{22}$. 


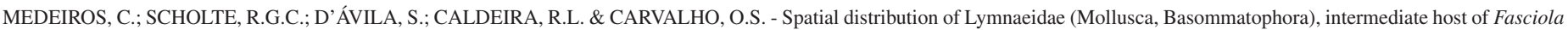
hepatica Linnaeus, 1758 (Trematoda, Digenea) in Brazil. Rev. Inst. Med. Trop. Sao Paulo, 56(3): 235-52, 2014.

Table 1

Distribution of Pseudosuccinea columella per municipalities and regions of Brazil

\begin{tabular}{|c|c|c|c|c|}
\hline Regions/States & Localities & Geographic coordinates & Species & $\begin{array}{c}\text { Articles/“SpeciesLink" } \\
\text { (CRIA) network }\end{array}$ \\
\hline \multicolumn{5}{|l|}{ 1) South } \\
\hline \multirow[t]{28}{*}{ Rio Grande do Sul } & Agudo & $29 \mathrm{~s} 38 / 53 w 15$ & P. columella & 1 \\
\hline & Arroio Grande & $32 \mathrm{~s} 14 / 53 \mathrm{w} 05$ & P. columella & 2 \\
\hline & Cachoeira do Sul & $30 \mathrm{~s} 02 / 52 \mathrm{w} 53$ & P. columella & 4 \\
\hline & Camaquã & $30 \mathrm{~s} 51 / 51 \mathrm{w} 48$ & P. columella ${ }^{+}$ & $4 ; 5 ; 6$ \\
\hline & Candiota & $31 \mathrm{~s} 35 / 53 \mathrm{w} 43$ & P. columella & MCP-Moluscos \\
\hline & Capão do Leão & $31 \mathrm{~s} 46 / 52 \mathrm{w} 30$ & P. columella & $4 ; 2$ \\
\hline & Dom Pedrito & $30 \mathrm{~s} 58 / 54 \mathrm{w} 40$ & P. columella ${ }^{+}$ & 4 \\
\hline & Eldorado do Sul & $29 \mathrm{~s} 50 / 51 w 18$ & P. columella & 81 \\
\hline & Gramado & $29 \mathrm{~s} 23 / 50 \mathrm{w} 52$ & P. columella & Fiocruz-CMM \\
\hline & Guaíba & $30 \mathrm{~s} 06 / 51 w 19$ & P. columella & 4 \\
\hline & Morro Reuter & $29 \mathrm{~s} 32 / 51 w 04$ & P. columella & 4 \\
\hline & Nova Petrópolis & $29 \mathrm{~s} 20 / 51 w 10$ & P. columella & 4 \\
\hline & Pelotas & $31 \mathrm{~s} 46 / 52 w 20$ & P. columella & 8; 9; Fiocruz-CMM \\
\hline & Porto Alegre & $30 \mathrm{~s} 01 / 51 w 13$ & P. columella & $11 ; 4$ \\
\hline & Rosário do Sul & $30 \mathrm{~s} 15 / 54 w 55$ & P. columella ${ }^{+}$ & 12 \\
\hline & Santa Maria & $29 \mathrm{~s} 41 / 53 \mathrm{w} 48$ & P. columella & 13 \\
\hline & Santa Vitória do Palmar & $33 \mathrm{~s} 31 / 53 w 22$ & P. columella & 4 \\
\hline & Santo Antônio da Patrulha & $29 \mathrm{~s} 49 / 50 w 30$ & P. columella & 84 \\
\hline & São Borja & $28 \mathrm{~s} 39 / 55 w 59$ & P. columella & 15 \\
\hline & São Leopoldo & $29 s 45 / 51 w 08$ & P. columella & MZUSP \\
\hline & São Martinho da Serra & $29 \mathrm{~s} 32 / 53 \mathrm{w} 51$ & P. columella & 13 \\
\hline & Sapucaia do Sul & $29 \mathrm{~s} 49 / 51 w 09$ & P. columella ${ }^{+}$ & 12 \\
\hline & Reserva ecológica do Taim & $32 \mathrm{~s} 29 / 52 \mathrm{w} 35$ & P. columella & 4 \\
\hline & Rio Pardo & $29 \mathrm{~s} 99 / 52 \mathrm{w} 35$ & P. columella & MCP-Moluscos \\
\hline & Taquara & $29 \mathrm{~s} 38 / 50 w 46$ & P. columella & $4 ; 87$ \\
\hline & Tramandaí & 29s59/50w07 & P. columella & 4 \\
\hline & Triunfo & $29 \mathrm{~s} 56 / 51 w 43$ & P. columella & 16; Fiocruz-CMM \\
\hline & Viamão & 30s04/51w01 & P. columella & 4 \\
\hline \multirow[t]{15}{*}{ Santa Catarina } & Araranguá & $28 \mathrm{~s} 56 / 49 w 28$ & P. columella & 4 \\
\hline & Biguaçu & $27 \mathrm{~s} 29 / 48 w 39$ & P. columella & $18 ; 19 ; 20$ \\
\hline & Blumenau & $26 \mathrm{~s} 54 / 49 w 04$ & P. columella & $18 ; 19 ; 20$ \\
\hline & Brusque & $27 \mathrm{~s} 05 / 48 \mathrm{w} 55$ & P. columella & $18 ; 19 ; 20$ \\
\hline & Camboriú & $27 \mathrm{~s} 01 / 48 w 39$ & P. columella & $18 ; 19 ; 20$ \\
\hline & Criciúma & $28 s 41 / 49 w 22$ & P. columella & $18 ; 19 ; 20$ \\
\hline & Florianópolis & $27 \mathrm{~s} 35 / 48 w 33$ & P. columella & $4 ; 17 ; 18 ; 19 ; 20$ \\
\hline & Itá & $27 \mathrm{~s} 29 / 52 \mathrm{w} 32$ & P. columella & MCP-Moluscos \\
\hline & Joinville & $26 \mathrm{~s} 17 / 48 \mathrm{w} 50$ & P. columella & 4 \\
\hline & Lages & $27 \mathrm{~s} 48 / 50 \mathrm{w} 19$ & P. columella & 4 \\
\hline & Lauro Müller & $28 \mathrm{~s} 23 / 49 w 24$ & P. columella & MZUSP \\
\hline & Nova Trento & $27 \mathrm{~s} 17 / 48 w 55$ & P. columella & $18 ; 19 ; 20$ \\
\hline & Palhoça & $27 \mathrm{~s} 38 / 48 w 40$ & P. columella & $18 ; 19 ; 20$ \\
\hline & São João do Sul & $29 \mathrm{~s} 13 / 49 w 48$ & P. columella & $18 ; 19 ; 20$ \\
\hline & Seara & $27 \mathrm{~s} 12 / 52 \mathrm{w} 19$ & P. columella & $\begin{array}{c}4 ; 24 \\
\text { Fiocruz-CMM }\end{array}$ \\
\hline
\end{tabular}


MEDEIROS, C.; SCHOLTE, R.G.C.; D’ÁVILA, S.; CALDEIRA, R.L. \& CARVALHO, O.S. - Spatial distribution of Lymnaeidae (Mollusca, Basommatophora), intermediate host of Fasciola hepatica Linnaeus, 1758 (Trematoda, Digenea) in Brazil. Rev. Inst. Med. Trop. Sao Paulo, 56(3): 235-52, 2014.

Table 1

Distribution of Pseudosuccinea columella per municipalities and regions of Brazil (cont.)

\begin{tabular}{|c|c|c|c|c|}
\hline Regions/States & Localities & Geographic coordinates & Species & $\begin{array}{l}\text { Articles/“SpeciesLink" } \\
\text { (CRIA) network }\end{array}$ \\
\hline \multirow[t]{2}{*}{ Santa Catarina } & Tubarão & $28 \mathrm{~s} 28 / 49 w 00$ & P. columella & $18 ; 19 ; 20$ \\
\hline & Urubici & $28 \mathrm{~s} 00 / 49 \mathrm{w} 35$ & P. columella & 25 \\
\hline \multirow[t]{15}{*}{ Paraná } & Antonina & $25 \mathrm{~s} 42 / 48 w 71$ & P. columella & 91 \\
\hline & Balsa Nova & $25 \mathrm{~s} 58 / 49 w 63$ & P. columella & 91 \\
\hline & Bocaiúva do Sul & $25 \mathrm{~s} 12 / 49 w 06$ & P. columella & $26 ; 91$ \\
\hline & Cambará & $23 \mathrm{~s} 02 / 50 \mathrm{w} 04$ & P. columella & Fiocruz-CMM \\
\hline & Campina Grande do Sul & $25 \mathrm{~s} 30 / 49 w 05$ & P. columella & 91 \\
\hline & Campo Largo & $25 \mathrm{~s} 45 / 49 w 53$ & P. columella & 91 \\
\hline & Contenda & $25 \mathrm{~s} 67 / 49 w 52$ & P. columella & 91 \\
\hline & Curitiba & $25 \mathrm{~s} 25 / 49 \mathrm{w} 16$ & P. columella & $\begin{array}{c}27 ; 4 ; 28 ; 24 ; 87 ; 52 ; 91 ; \\
\text { Fiocruz-CMM } \\
\text { MZUSP }\end{array}$ \\
\hline & Dionísio Cerqueira (Barracão) & $26 \mathrm{~s} 15 / 53 w 38$ & P. columella & 4 \\
\hline & Morretes & $25 \mathrm{~s} 28 / 48 w 49$ & P. columella & $4 ; 91$ \\
\hline & Paranaguá & $25 \mathrm{~s} 30 / 48 w 30$ & P. columella & MZUSP \\
\hline & Quatro Barras & $25 \mathrm{~s} 36 / 49 w 07$ & P. columella & 91 \\
\hline & Rio Branco do Sul & $25 \mathrm{~s} 19 / 49 w 31$ & P. columella & 91 \\
\hline & São José dos Pinhais & $25 \mathrm{~s} 32 / 49 w 12$ & P. columella & $87 ; 91$ \\
\hline & Tunas do Paraná & $24 \mathrm{~s} 58 / 49 w 05$ & P. columella & 26; MZUSP \\
\hline \multicolumn{5}{|l|}{ 2) Southeast } \\
\hline \multirow[t]{25}{*}{ São Paulo } & Águas de Santa Bárbara & $22 \mathrm{~s} 52 / 49 w 14$ & P. columella & 29 \\
\hline & Altinópolis & $21 \mathrm{~s} 04 / 47 \mathrm{w} 22$ & P. columella & 30 \\
\hline & Álvares Machado & $22 \mathrm{~s} 04 / 51 w 28$ & P. columella & 31 \\
\hline & Americana & $22 s 44 / 47 w 19$ & P. columella & 80 \\
\hline & Américo Brasiliense & $21 \mathrm{~s} 43 / 48 w 06$ & P. columella & 30 \\
\hline & Angatuba & 23 s29/48w24 & P. columella & 29 \\
\hline & Anhembi & $22 s 47 / 48 w 07$ & P. columella & 29 \\
\hline & Araçoiaba da Serra & $23 \mathrm{~s} 30 / 47 w 36$ & P. columella & 29 \\
\hline & Aramina & $20 \mathrm{~s} 05 / 47 w 47$ & P. columella & 30 \\
\hline & Arandu & $23 \mathrm{~s} 08 / 49 \mathrm{w} 03$ & P. columella & 29 \\
\hline & Araraquara & $21 \mathrm{~s} 47 / 48 w 10$ & P. columella & 30 \\
\hline & Areiópolis & $22 \mathrm{~s} 40 / 48 w 39$ & P. columella & 29 \\
\hline & Atibaia & $23 \mathrm{~s} 69 / 46 w 33$ & P. columella & 80 \\
\hline & Avanhandava & $21 \mathrm{~s} 27 / 49 w 56$ & P. columella & MZUSP \\
\hline & Avaré & $21 \mathrm{~s} 54 / 49 w 21$ & P. columella & 29 \\
\hline & Barão de Antonina & $23 s 37 / 49 w 33$ & P. columella & 29 \\
\hline & Barra Bonita & $22 \mathrm{~s} 29 / 48 w 33$ & P. columella & 33 \\
\hline & Barretos & 20 s33/48w34 & P. columella & 30 \\
\hline & Batatais & $20 \mathrm{~s} 53 / 47 w 35$ & P. columella & 30 \\
\hline & Bebedouro & $20 \mathrm{~s} 56 / 48 w 28$ & P. columella & 30 \\
\hline & Boa Esperança do Sul & $21 \mathrm{~s} 59 / 48 w 23$ & P. columella & 30 \\
\hline & Bofete & $23 \mathrm{~s} 05 / 48 \mathrm{w} 15$ & P. columella & 29 \\
\hline & Boituva & $23 \mathrm{~s} 17 / 47 \mathrm{w} 40$ & P. columella & 29 \\
\hline & Borborema & $21 \mathrm{~s} 37 / 49 w 04$ & P. columella & 30 \\
\hline & Botucatu & $22 \mathrm{~s} 53 / 48 w 26$ & P. columella & 29 \\
\hline
\end{tabular}




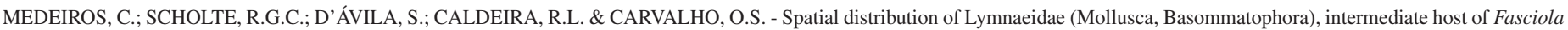
hepatica Linnaeus, 1758 (Trematoda, Digenea) in Brazil. Rev. Inst. Med. Trop. Sao Paulo, 56(3): 235-52, 2014.

Table 1

Distribution of Pseudosuccinea columella per municipalities and regions of Brazil (cont.)

\begin{tabular}{|c|c|c|c|c|}
\hline Regions/States & Localities & Geographic coordinates & Species & $\begin{array}{l}\text { Articles/“SpeciesLink" } \\
\text { (CRIA) network }\end{array}$ \\
\hline \multirow{44}{*}{ São Paulo } & Brodowski & 20s59/47w39 & P. columella & 30 \\
\hline & Brotas & $22 \mathrm{~s} 17 / 48 w 07$ & P. columella & MZUSP \\
\hline & Buri & $23 \mathrm{~s} 47 / 48 w 35$ & P. columella & 29 \\
\hline & Cabreúva & $23 \mathrm{~s} 18 / 47 w 07$ & P. columella & 29 \\
\hline & Caçapava & $23 \mathrm{~s} 05 / 45 w 42$ & P. columella & $4 ; 80$ \\
\hline & Caiuá & 21s49/51w59 & P. columella & 31 \\
\hline & Cajurú & $21 \mathrm{~s} 16 / 47 \mathrm{w} 18$ & P. columella & 30 \\
\hline & Campinas & $22 \mathrm{~s} 53 / 47 \mathrm{w} 05$ & P. columella & $34 ; 4 ; 35 ; 36 ; 80$ \\
\hline & Cândido Rodrigues & 21s19/48w37 & P. columella & 30 \\
\hline & Capão Bonito & $24 \mathrm{~s} 00 / 48 \mathrm{w} 20$ & P. columella & 29 \\
\hline & Capela do Alto & $23 \mathrm{~s} 27 / 47 \mathrm{w} 44$ & P. columella & 29 \\
\hline & Caraguatatuba & $23 \mathrm{~s} 27 / 45 \mathrm{w} 24$ & P. columella & MZUSP \\
\hline & Cássia dos Coqueiros & $21 \mathrm{~s} 16 / 47 \mathrm{w} 10$ & P. columella & 30 \\
\hline & Catiguá & $21 \mathrm{~s} 03 / 49 w 03$ & P. columella & 37 \\
\hline & Cerqueira Cesar & $23 \mathrm{~s} 01 / 49 w 09$ & P. columella & 29 \\
\hline & Cerquilho & $23 \mathrm{~s} 09 / 47 \mathrm{w} 44$ & P. columella & 29 \\
\hline & Cesário Lange & $23 \mathrm{~s} 13 / 47 w 57$ & P. columella & 29 \\
\hline & Colina & $20 s 43 / 48 w 32$ & P. columella & 30 \\
\hline & Colômbia & $20 \mathrm{~s} 10 / 48 w 41$ & P. columella & 30 \\
\hline & Conchas & $23 \mathrm{~s} 01 / 48 \mathrm{w} 00$ & P. columella & 29 \\
\hline & Coronel Macedo & $23 \mathrm{~s} 38 / 49 w 18$ & P. columella & 29 \\
\hline & Cravinhos & $21 \mathrm{~s} 20 / 47 w 43$ & P. columella & 30 \\
\hline & Cristais Paulista & $20 \mathrm{~s} 23 / 47 w 25$ & P. columella & 30 \\
\hline & Descalvado & $21 \mathrm{~s} 54 / 47 w 37$ & P. columella & 30 \\
\hline & Dourado & $22 \mathrm{~s} 06 / 48 w 18$ & P. columella & 30 \\
\hline & Eldorado & $24 \mathrm{~s} 30 / 48 w 05$ & P. columella ${ }^{+}$ & 41 \\
\hline & Estrela do Norte & $22 \mathrm{~s} 29 / 51 w 39$ & P. columella & 31 \\
\hline & Fernando Prestes & $21 \mathrm{~s} 15 / 48 \mathrm{w} 41$ & P. columella & 30 \\
\hline & Franca & $20 \mathrm{~s} 31 / 47 w 23$ & P. columella & 30 \\
\hline & Guaíra & $20 \mathrm{~s} 19 / 48 w 19$ & P. columella & 30 \\
\hline & Guapiaçú & $20 s 47 / 49 w 13$ & P. columella & 37 \\
\hline & Guapiara & $24 \mathrm{~s} 10 / 48 \mathrm{w} 32$ & P. columella & 29 \\
\hline & Guará & $20 \mathrm{~s} 25 / 47 w 49$ & P. columella & 30 \\
\hline & Guaraci & 20 s29/48w56 & P. columella & 37 \\
\hline & Guareí & $23 \mathrm{~s} 22 / 48 w 10$ & P. columella & 29 \\
\hline & Guarujá & $23 \mathrm{~s} 58 / 46 w 15$ & P. columella & MZUSP \\
\hline & Ibaté & 21s57/47w59 & P. columella & 30 \\
\hline & Ibirá & $21 \mathrm{~s} 04 / 49 w 14$ & P. columella & 37 \\
\hline & Ibitinga & $21 \mathrm{~s} 45 / 48 w 49$ & P. columella & 30 \\
\hline & Ibiúna & $23 \mathrm{~s} 38 / 47 \mathrm{w} 13$ & P. columella & 29 \\
\hline & Iepê & $22 \mathrm{~s} 38 / 51 w 06$ & P. columella & 31 \\
\hline & Igarapava & $20 \mathrm{~s} 02 / 47 w 44$ & P. columella & 30; Fiocruz-CMM \\
\hline & Indiana & $22 \mathrm{~s} 08 / 51 w 15$ & P. columella & 31 \\
\hline & Iperó & $23 \mathrm{~s} 21 / 47 \mathrm{w} 42$ & P. columella & 29 \\
\hline
\end{tabular}


MEDEIROS, C.; SCHOLTE, R.G.C.; D’ÁVILA, S.; CALDEIRA, R.L. \& CARVALHO, O.S. - Spatial distribution of Lymnaeidae (Mollusca, Basommatophora), intermediate host of Fasciola hepatica Linnaeus, 1758 (Trematoda, Digenea) in Brazil. Rev. Inst. Med. Trop. Sao Paulo, 56(3): 235-52, 2014.

Table 1

Distribution of Pseudosuccinea columella per municipalities and regions of Brazil (cont.)

\begin{tabular}{|c|c|c|c|c|}
\hline Regions/States & Localities & Geographic coordinates & Species & $\begin{array}{c}\text { Articles/“SpeciesLink" } \\
\text { (CRIA) network }\end{array}$ \\
\hline \multirow[t]{44}{*}{ São Paulo } & Irapuã & $21 \mathrm{~s} 17 / 49 w 24$ & P. columella & 37 \\
\hline & Itaberá & $23 \mathrm{~s} 51 / 49208$ & P. columella & 29 \\
\hline & Itaí & $23 \mathrm{~s} 24 / 49 w 05$ & P. columella & 29 \\
\hline & Itapetininga & $23 \mathrm{~s} 36 / 48 w 03$ & P. columella & 29 \\
\hline & Itapeva & $23 \mathrm{~s} 58 / 48 w 52$ & P. columella & 29 \\
\hline & Itápolis & $21 \mathrm{~s} 35 / 48 w 48$ & P. columella & 30 \\
\hline & Itaporanga & $23 \mathrm{~s} 42 / 48 w 29$ & P. columella & 29 \\
\hline & Itararé & $24 \mathrm{~s} 06 / 49 w 20$ & P. columella & 29 \\
\hline & Itariri & $24 \mathrm{~s} 17 / 47 \mathrm{w} 03$ & P. columella & $4 ; 32$ \\
\hline & Itatinga & $23 \mathrm{~s} 06 / 48 w 36$ & P. columella & 29 \\
\hline & Itu & $23 \mathrm{~s} 15 / 47 \mathrm{w} 17$ & P. columella & 29 \\
\hline & Itupeva & $23 \mathrm{~s} 08 / 47 \mathrm{w} 04$ & P. columella & MZUSP \\
\hline & Ituverava & $20 s 20 / 47 w 47$ & P. columella & 30 \\
\hline & Jaboticabal & $21 \mathrm{~s} 15 / 48 \mathrm{w} 18$ & P. columella & 30 \\
\hline & Jaguariúna & $22 \mathrm{~s} 42 / 46 w 59$ & P. columella & 36 \\
\hline & Jardinópolis & $21 \mathrm{~s} 01 / 47 \mathrm{w} 45$ & P. columella & 30 \\
\hline & João Ramalho & $22 \mathrm{~s} 15 / 50 w 46$ & P. columella & 31 \\
\hline & Junqueirópolis & $21 \mathrm{~s} 31 / 51 w 26$ & P. columella & 31 \\
\hline & Louveira & $23 \mathrm{~s} 05 / 46 w 58$ & P. columella & 36 \\
\hline & Luis Antônio & $21 \mathrm{~s} 33 / 47 w 42$ & P. columella & 30 \\
\hline & Mairinque & $23 \mathrm{~s} 32 / 47 w 11$ & P. columella & 29 \\
\hline & Marinópolis & $20 \mathrm{~s} 26 / 50 w 49$ & P. columella & 37 \\
\hline & Martinópolis & $22 \mathrm{~s} 08 / 51 w 10$ & P. columella & 31 \\
\hline & Matão & $21 \mathrm{~s} 36 / 48 w 21$ & P. columella & 30 \\
\hline & Miguelópolis & $20 \mathrm{~s} 10 / 48 w 01$ & P. columella & 30 \\
\hline & Miracatu & $24 \mathrm{~s} 16 / 47 w 27$ & P. columella ${ }^{+}$ & $36 ; 41$ \\
\hline & Monte Alto & $21 \mathrm{~s} 15 / 48 \mathrm{w} 29$ & P. columella & 30 \\
\hline & Monte Aprazível & $20 \mathrm{~s} 45 / 49 w 42$ & P. columella & 37 \\
\hline & Monte Azul Paulista & $20 \mathrm{~s} 54 / 48 w 38$ & P. columella & 30 \\
\hline & Morro Agudo & $20 s 43 / 48 w 03$ & P. columella & 30 \\
\hline & Narandiba & $22 \mathrm{~s} 24 / 51 w 31$ & P. columella & 31 \\
\hline & Nova Europa & $21 \mathrm{~s} 46 / 48 w 33$ & P. columella & 30 \\
\hline & Nova Granada & $20 s 31 / 49 w 18$ & P. columella & 37 \\
\hline & Novo Horizonte & $21 \mathrm{~s} 28 / 49 \mathrm{w} 13$ & P. columella & 37 \\
\hline & Olímpia & $20 s 44 / 48 w 54$ & P. columella & 37 \\
\hline & Orlândia & $20 \mathrm{~s} 43 / 47$ w53 & P. columella & 30 \\
\hline & Oswaldo Cruz & $23 s 37 / 46 w 34$ & P. columella & 31 \\
\hline & Palestina & $20 \mathrm{~s} 21 / 49 w 25$ & P. columella & 37 \\
\hline & Palmital & $22 \mathrm{~s} 46 / 50 \mathrm{w} 12$ & P. columella & Fiocruz-CMM \\
\hline & Panorama & 21s20/51w51 & P. columella & 31 \\
\hline & Paraíso & $20 \mathrm{~s} 59 / 48 \mathrm{w} 46$ & P. columella & 37 \\
\hline & Paranapanema & $23 \mathrm{~s} 23 / 48 w 43$ & P. columella & 29 \\
\hline & Parapuã & $21 \mathrm{~s} 46 / 50 w 47$ & P. columella & 31 \\
\hline & Pardinho & $23 \mathrm{~s} 04 / 48 w 22$ & P. columella & 29 \\
\hline
\end{tabular}




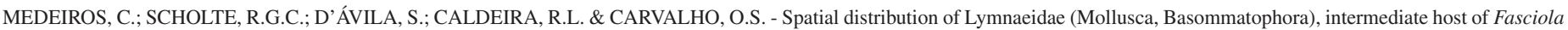
hepatica Linnaeus, 1758 (Trematoda, Digenea) in Brazil. Rev. Inst. Med. Trop. Sao Paulo, 56(3): 235-52, 2014.

Table 1

Distribution of Pseudosuccinea columella per municipalities and regions of Brazil (cont.)

\begin{tabular}{|c|c|c|c|c|}
\hline Regions/States & Localities & Geographic coordinates & Species & $\begin{array}{l}\text { Articles/“SpeciesLink" } \\
\text { (CRIA) network }\end{array}$ \\
\hline \multirow{44}{*}{ São Paulo } & Patrocínio Paulista & 20 s37/47w16 & P. columella & 30 \\
\hline & Pedregulho & $20 \mathrm{~s} 14 / 47$ w28 & P. columella & 30 \\
\hline & Pereiras & $23 \mathrm{~s} 04 / 47 w 58$ & P. columella & 29 \\
\hline & Piedade & $23 \mathrm{~s} 41 / 47 w 25$ & P. columella & 29 \\
\hline & Pilar do Sul & $23 \mathrm{~s} 48 / 47 \mathrm{w} 43$ & P. columella & 29 \\
\hline & Piquerobi & $21 \mathrm{~s} 52 / 51 w 43$ & P. columella & 31 \\
\hline & Piquete & $22 \mathrm{~s} 36 / 45 w 10$ & P. columella ${ }^{+}$ & $38 ; 39 ; 75 ;$ MZUSP \\
\hline & Piracicaba & $22 s 43 / 47 w 39$ & P. columella & 36 \\
\hline & Pirangi & 21s09/48w39 & P. columella & 30 \\
\hline & Pirassununga & $21 \mathrm{~s} 59 / 47 \mathrm{w} 25$ & P. columella & $4 ; 36 ; 80$ \\
\hline & Pitangueiras & $20 \mathrm{~s} 59 / 48 w 12$ & P. columella & 30 \\
\hline & Planalto & $21 \mathrm{~s} 21 / 49 \mathrm{w} 55$ & P. columella & 37 \\
\hline & Pontal & 20s59/48w02 & P. columella & 30 \\
\hline & Porangaba & $20 \mathrm{~s} 54 / 47$ w53 & P. columella & 29 \\
\hline & Porto Feliz & $23 \mathrm{~s} 12 / 47 \mathrm{w} 31$ & P. columella & 29 \\
\hline & Pradópolis & $21 \mathrm{~s} 21 / 48 w 03$ & P. columella & 30 \\
\hline & Presidente Bernardes & $22 \mathrm{~s} 00 / 51 w 33$ & P. columella & 31 \\
\hline & Rancharia & $22 \mathrm{~s} 13 / 50 w 53$ & P. columella & 31 \\
\hline & Redenção da Serra & $23 \mathrm{~s} 13 / 45 w 32$ & P. columella & 38 \\
\hline & Regente Feijó & $22 \mathrm{~s} 13 / 51 w 18$ & P. columella & 31 \\
\hline & Registro & $24 \mathrm{~s} 29 / 47 \mathrm{w} 51$ & P. columella & 36 \\
\hline & Restinga & $20 \mathrm{~s} 34 / 47 w 29$ & P. columella & 30 \\
\hline & Ribeirão Bonito & $20 \mathrm{~s} 02 / 48 w 10$ & P. columella & 30 \\
\hline & Ribeirão Branco & $24 \mathrm{~s} 11 / 48 w 46$ & P. columella & 29 \\
\hline & Ribeirão Preto & $21 \mathrm{~s} 10 / 47 \mathrm{w} 48$ & P. columella & 4; 30; MZUSP \\
\hline & Rincão & 21s34/48w04 & P. columella & 30 \\
\hline & Rinópolis & $21 \mathrm{~s} 43 / 50 \mathrm{w} 43$ & P. columella & 31 \\
\hline & Rio Claro & $22 \mathrm{~s} 23 / 47 w 33$ & P. columella & 4 \\
\hline & Riversul & $23 \mathrm{~s} 48 / 49 w 24$ & P. columella & 29 \\
\hline & Rubinéia & $20 \mathrm{~s} 10 / 50 w 59$ & P. columella & 37 \\
\hline & Sales & $21 \mathrm{~s} 20 / 49 w 30$ & P. columella & 37 \\
\hline & Sales Oliveira & $20 \mathrm{~s} 46 / 47 w 56$ & P. columella & 30 \\
\hline & Salesópolis & $23 \mathrm{~s} 31 / 45 w 50$ & P. columella & 78; MZUSP \\
\hline & Salto & $23 \mathrm{~s} 11 / 47 w 17$ & P. columella & 29 \\
\hline & Salto de Pirapora & $23 \mathrm{~s} 38 / 47 w 34$ & P. columella & 29 \\
\hline & Sandovalina & $22 \mathrm{~s} 26 / 51 w 46$ & P. columella & 31 \\
\hline & Santa Adélia & $21 \mathrm{~s} 14 / 48 w 47$ & P. columella & 37 \\
\hline & Santa Mercedes & $21 \mathrm{~s} 20 / 51 w 45$ & P. columella & 31 \\
\hline & Santa Rita do Passa Quatro & $21 \mathrm{~s} 42 / 47 \mathrm{w} 28$ & P. columella & 30 \\
\hline & Santa Rosa do Viterbo & $21 \mathrm{~s} 28 / 47 \mathrm{w} 21$ & P. columella & 30 \\
\hline & Santo Anastácio & $21 \mathrm{~s} 57 / 51 \mathrm{w} 38$ & P. columella & 31 \\
\hline & Santo Expedito & $21 \mathrm{~s} 50 / 51 w 23$ & P. columella & 31 \\
\hline & São Carlos & $21 \mathrm{~s} 59 / 47$ w53 & P. columella & 30 \\
\hline & São Joaquim da Barra & $20 \mathrm{~s} 34 / 47$ w5 2 & P. columella & 30 \\
\hline
\end{tabular}


MEDEIROS, C.; SCHOLTE, R.G.C.; D’ÁVILA, S.; CALDEIRA, R.L. \& CARVALHO, O.S. - Spatial distribution of Lymnaeidae (Mollusca, Basommatophora), intermediate host of Fasciola hepatica Linnaeus, 1758 (Trematoda, Digenea) in Brazil. Rev. Inst. Med. Trop. Sao Paulo, 56(3): 235-52, 2014.

Table 1

Distribution of Pseudosuccinea columella per municipalities and regions of Brazil (cont.)

\begin{tabular}{|c|c|c|c|c|}
\hline Regions/States & Localities & Geographic coordinates & Species & $\begin{array}{c}\text { Articles/“SpeciesLink" } \\
\text { (CRIA) network }\end{array}$ \\
\hline \multirow[t]{26}{*}{ São Paulo } & São José do Rio Preto & $20 s 40 / 49 w 17$ & P. columella & 37 \\
\hline & São José dos Campos & $23 \mathrm{~s} 11 / 45 w 52$ & P. columella & 4 \\
\hline & São Manoel & $22 s 43 / 47 w 18$ & P. columella & 29 \\
\hline & São Miguel Arcanjo & $23 \mathrm{~s} 52 / 47 \mathrm{w} 59$ & P. columella & 29 \\
\hline & São Paulo & $23 s 32 / 46 w 38$ & P. columella & 4; MZUSP \\
\hline & São Roque & $23 \mathrm{~s} 31 / 47 w 08$ & P. columella & 29 \\
\hline & São Simão & $21 \mathrm{~s} 28 / 47 w 33$ & P. columella & 30 \\
\hline & São Vicente & $23 \mathrm{~s} 57 / 46 w 22$ & P. columella & MZUSP \\
\hline & Sarapuí & $23 \mathrm{~s} 37 / 47 w 49$ & P. columella & 29 \\
\hline & Serrana & $21 \mathrm{~s} 12 / 47 \mathrm{w} 36$ & P. columella & 30 \\
\hline & Sertãozinho & $21 \mathrm{~s} 06 / 47 \mathrm{w} 59$ & P. columella & 30 \\
\hline & Sorocaba & $23 \mathrm{~s} 28 / 47 w 25$ & P. columella & 29 \\
\hline & Tabatinga & $21 \mathrm{~s} 42 / 48 \mathrm{w} 40$ & P. columella & 30 \\
\hline & Taiaçu & $21 \mathrm{~s} 08 / 48 w 30$ & P. columella & 30 \\
\hline & Taiuva & $21 \mathrm{~s} 06 / 48 w 26$ & P. columella & 30 \\
\hline & Tapuaí & $21 \mathrm{~s} 54 / 49 w 21$ & P. columella & 29 \\
\hline & Taquaritinga & $21 \mathrm{~s} 24 / 48 w 29$ & P. columella & 30 \\
\hline & Taquarituba & $23 \mathrm{~s} 31 / 49 w 14$ & P. columella & 29 \\
\hline & Tarabaí & $22 \mathrm{~s} 17 / 51 w 33$ & P. columella & 31 \\
\hline & Tatuí & $23 \mathrm{~s} 21 / 47 \mathrm{w} 50$ & P. columella & 29 \\
\hline & Taubaté & $23 \mathrm{~s} 00 / 45 w 33$ & P. columella & $4 ; 80$ \\
\hline & Terra Roxa & $20 s 46 / 48 w 19$ & P. columella & 30 \\
\hline & Tietê & $23 \mathrm{~s} 05 / 47 \mathrm{w} 42$ & P. columella & 29 \\
\hline & Urupês & $21 \mathrm{~s} 08 / 49 w 15$ & P. columella & 37 \\
\hline & Vista Alegre do Alto & $21 \mathrm{~s} 09 / 48 w 38$ & P. columella & 30 \\
\hline & Votorantim & $23 \mathrm{~s} 32 / 47 w 26$ & P. columella & 29 \\
\hline \multirow[t]{18}{*}{ Rio de Janeiro } & Angra dos Reis & $23 \mathrm{~s} 00 / 44 w 19$ & P. columella & 42 \\
\hline & Aperibé & $21 \mathrm{~s} 37 / 42 w 06$ & P. columella & 43 \\
\hline & Araruama & $22 \mathrm{~s} 50 / 42 \mathrm{w} 20$ & P. columella & 44 \\
\hline & Areal & $22 \mathrm{~s} 13 / 43 \mathrm{w} 06$ & P. columella & 45 \\
\hline & Barra do Piraí & $22 \mathrm{~s} 27 / 43 \mathrm{w} 48$ & P. columella & 42 \\
\hline & Barra Mansa & $22 \mathrm{~s} 32 / 44 \mathrm{w} 10$ & P. columella & 42 \\
\hline & Bom Jardim & $22 \mathrm{~s} 09 / 42 \mathrm{w} 25$ & P. columella & 45 \\
\hline & Bom Jesus de Itabapoana & $21 \mathrm{~s} 07 / 41 \mathrm{w} 40$ & P. columella & 43 \\
\hline & Cachoeiras de Macacu & $22 \mathrm{~s} 31 / 42 \mathrm{w} 42$ & P. columella ${ }^{+}$ & $46 ; 76$ \\
\hline & Cambuci & $21 \mathrm{~s} 33 / 41 w 54$ & P. columella & 43 \\
\hline & Campos dos Goytacazes & $21 \mathrm{~s} 45 / 41 w 20$ & P. columella ${ }^{+}$ & $47 ; 48 ; 49$ \\
\hline & Cantagalo & $21 \mathrm{~s} 58 / 42 w 22$ & P. columella & 45 \\
\hline & Cardoso Moreira & $21 \mathrm{~s} 38 / 41 w 45$ & P. columella & 49 \\
\hline & Carmo & $21 \mathrm{~s} 54 / 42 w 36$ & P. columella & 45 \\
\hline & Casimiro de Abreu & $22 \mathrm{~s} 27 / 42 w 12$ & P. columella & 44 \\
\hline & Comendador Levy Gasparian & $22 \mathrm{~s} 00 / 43 \mathrm{w} 12$ & P. columella & 45 \\
\hline & Conceição de Macabu & $22 \mathrm{~s} 03 / 41 w 51$ & P. columella & 49 \\
\hline & Cordeiro & $22 \mathrm{~s} 00 / 42 \mathrm{w} 21$ & P. columella & 45 \\
\hline
\end{tabular}




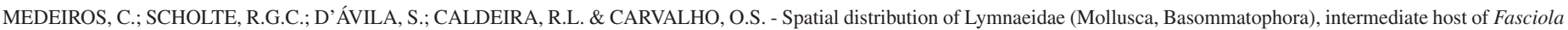
hepatica Linnaeus, 1758 (Trematoda, Digenea) in Brazil. Rev. Inst. Med. Trop. Sao Paulo, 56(3): 235-52, 2014.

Table 1

Distribution of Pseudosuccinea columella per municipalities and regions of Brazil (cont.)

\begin{tabular}{|c|c|c|c|c|}
\hline Regions/States & Localities & Geographic coordinates & Species & $\begin{array}{l}\text { Articles/“SpeciesLink" } \\
\text { (CRIA) network }\end{array}$ \\
\hline \multirow[t]{44}{*}{ Rio de Janeiro } & Duas Barras & $22 \mathrm{~s} 02 / 42 \mathrm{w} 31$ & P. columella & 45 \\
\hline & Engenheiro Paulo de Frontin & $22 \mathrm{~s} 33 / 43 w 41$ & P. columella & 46 \\
\hline & Guapimirim & $22 \mathrm{~s} 29 / 42 w 59$ & P. columella & 50 \\
\hline & Itaboraí & $22 \mathrm{~s} 43 / 42 w 51$ & P. columella & 46 \\
\hline & Itaguaí & $22 \mathrm{~s} 51 / 43 w 46$ & P. columella ${ }^{+}$ & $46 ; 51 ; 85$ \\
\hline & Italva & $21 \mathrm{~s} 27 / 41 w 40$ & P. columella & 43 \\
\hline & Itaocara & $21 \mathrm{~s} 38 / 42 \mathrm{w} 04$ & P. columella & 43 \\
\hline & Itaperuna & $21 \mathrm{~s} 10 / 41 \mathrm{w} 53$ & P. columella & 43 \\
\hline & Itatiaia & $22 \mathrm{~s} 25 / 44 w 33$ & P. columella & 42 \\
\hline & Japeri & $22 \mathrm{~s} 38 / 43 w 39$ & P. columella & 46 \\
\hline & Laje do Muriaé & $21 \mathrm{~s} 11 / 42 w 07$ & P. columella & 43 \\
\hline & Macaé & $22 \mathrm{~s} 21 / 41 \mathrm{w} 46$ & P. columella & 49 \\
\hline & Magé & $22 \mathrm{~s} 38 / 43 w 02$ & P. columella & $50 ; 51$ \\
\hline & Mangaratiba & $22 \mathrm{~s} 56 / 44 w 02$ & P. columella & 46 \\
\hline & Maricá & $22 \mathrm{~s} 54 / 42 w 49$ & P. columella & 46 \\
\hline & Mendes & $22 \mathrm{~s} 31 / 43 w 44$ & P. columella & 46 \\
\hline & Miguel Pereira & $22 \mathrm{~s} 26 / 43 \mathrm{w} 28$ & P. columella & 46 \\
\hline & Miracema & $21 \mathrm{~s} 22 / 42 \mathrm{w} 12$ & P. columella & 43 \\
\hline & Natividade & $21 \mathrm{~s} 01 / 41 \mathrm{w} 58$ & P. columella & 43 \\
\hline & Niterói & $22 \mathrm{~s} 52 / 43 w 07$ & P. columella & $46 ; 53$ \\
\hline & Nova Friburgo & $22 \mathrm{~s} 15 / 42 w 31$ & P. columella & 45 \\
\hline & Nova Iguaçu & $22 s 44 / 43 w 27$ & P. columella & 46 \\
\hline & Paracambi & $22 \mathrm{~s} 29 / 43 \mathrm{w} 41$ & P. columella & 46 \\
\hline & Paraíba do Sul & $22 \mathrm{~s} 08 / 43 w 16$ & P. columella & 45 \\
\hline & Parati & $23 \mathrm{~s} 10 / 44 w 43$ & P. columella & 42 \\
\hline & Paty dos Alferes & $22 \mathrm{~s} 25 / 43 \mathrm{w} 25$ & P. columella & 46 \\
\hline & Petrópolis & $22 \mathrm{~s} 21 / 43 w 08$ & P. columella & 50 \\
\hline & Pinheiral & $22 \mathrm{~s} 31 / 44 w 00$ & P. columella & 42 \\
\hline & Piraí & $22 \mathrm{~s} 30 / 44 w 00$ & P. columella & 42 \\
\hline & Porciúncula & 20s57/42w02 & P. columella & 43 \\
\hline & Quatis & $22 s 40 / 44 w 27$ & P. columella & 42 \\
\hline & Resende & $22 \mathrm{~s} 27 / 44 w 26$ & P. columella & 42 \\
\hline & Rio Bonito & $22 \mathrm{~s} 40 / 42 w 37$ & P. columella & 46 \\
\hline & Rio Claro & $22 \mathrm{~s} 72 / 44 w 13$ & P. columella & 42 \\
\hline & Rio das Flores & $22 \mathrm{~s} 15 / 43 \mathrm{w} 58$ & P. columella & 42 \\
\hline & Rio das Ostras & $22 \mathrm{~s} 29 / 42 w 59$ & P. columella & 44 \\
\hline & Rio de Janeiro & $22 \mathrm{~s} 53 / 43 \mathrm{w} 12$ & P. columella & $4 ; 46 ; 54 ; 86$ \\
\hline & Santa Maria Madalena & 21s97/41w99 & P. columella & 45 \\
\hline & Santo Antônio de Pádua & $21 \mathrm{~s} 53 / 42 \mathrm{w} 18$ & P. columella & 43 \\
\hline & São Fidélis & $21 \mathrm{~s} 37 / 41 \mathrm{w} 44$ & P. columella & 49 \\
\hline & São Gonçalo & $22 \mathrm{~s} 83 / 43 w 05$ & P. columella & $46 ; 51$ \\
\hline & São João de Meriti & $22 \mathrm{~s} 80 / 43 w 37$ & P. columella & 46 \\
\hline & São José de Ubá & 21 s36/41w93 & P. columella & 43 \\
\hline & São José do Vale do Rio Preto & $22 \mathrm{~s} 08 / 42 \mathrm{w} 55$ & P. columella & 50 \\
\hline
\end{tabular}


MEDEIROS, C.; SCHOLTE, R.G.C.; D’ÁVILA, S.; CALDEIRA, R.L. \& CARVALHO, O.S. - Spatial distribution of Lymnaeidae (Mollusca, Basommatophora), intermediate host of Fasciola hepatica Linnaeus, 1758 (Trematoda, Digenea) in Brazil. Rev. Inst. Med. Trop. Sao Paulo, 56(3): 235-52, 2014.

Table 1

Distribution of Pseudosuccinea columella per municipalities and regions of Brazil (cont.)

\begin{tabular}{|c|c|c|c|c|}
\hline Regions/States & Localities & Geographic coordinates & Species & $\begin{array}{c}\text { Articles/“SpeciesLink" } \\
\text { (CRIA) network }\end{array}$ \\
\hline \multirow[t]{14}{*}{ Rio de Janeiro } & São Sebastião do Alto & $21 \mathrm{~s} 95 / 42 w 14$ & P. columella & 45 \\
\hline & Sapucaia & 21s99/42w90 & P. columella & 45 \\
\hline & Saquarema & $22 s 93 / 42 w 49$ & P. columella & 44 \\
\hline & Seropédica & $22 \mathrm{~s} 51 / 43 \mathrm{w} 45$ & P. columella & 56; 89; Fiocruz-CMM \\
\hline & Silva Jardim & $22 \mathrm{~s} 37 / 42 w 23$ & P. columella & 44 \\
\hline & Sumidouro & $22 \mathrm{~s} 04 / 42 w 67$ & P. columella & 45 \\
\hline & Tamoios & $22 \mathrm{~s} 72 / 42 \mathrm{w} 01$ & P. columella & 44 \\
\hline & Teresópolis & $22 \mathrm{~s} 23 / 42 w 58$ & P. columella & 50 \\
\hline & Trajano de Morais & $22 \mathrm{~s} 07 / 42 \mathrm{w} 06$ & P. columella & 45 \\
\hline & Três Rios & $22 \mathrm{~s} 05 / 43 \mathrm{w} 12$ & P. columella & $4 ; 45$ \\
\hline & Valença & $22 \mathrm{~s} 24 / 43 \mathrm{w} 70$ & P. columella & 42 \\
\hline & Varre-Sai & 20 s93/41w87 & P. columella & 43 \\
\hline & Vassouras & $22 s 40 / 43 w 66$ & P. columella & $11 ; 46$ \\
\hline & Volta Redonda & $22 \mathrm{~s} 52 / 44 \mathrm{w} 10$ & P. columella & 42 \\
\hline \multirow[t]{26}{*}{ Minas Gerais } & Alfenas & $21 \mathrm{~s} 25 / 45 \mathrm{w} 56$ & P. columella & Fiocruz-CMM \\
\hline & Barbacena & $21 \mathrm{~s} 13 / 43 \mathrm{w} 46$ & P. columella & 4 \\
\hline & Belo Horizonte & $19 \mathrm{~s} 55 / 43 \mathrm{w} 56$ & P. columella & $\begin{array}{c}\text { 11; 4; 58; 59; 23; } 24 \\
\text { Fiocruz-CMM } \\
\text { INPA-Mollusca }\end{array}$ \\
\hline & Betim & 19s58/44w04 & P. columella & $\begin{array}{c}4 ; 59 ; 61 \\
\text { Fiocruz-CMM }\end{array}$ \\
\hline & Bicas & $21 \mathrm{~s} 43 / 43 \mathrm{w} 04$ & P. columella & 61 \\
\hline & Brasópolis & $22 \mathrm{~s} 27 / 45 w 36$ & P. columella & 61 \\
\hline & Cachoeira de Minas & $22 \mathrm{~s} 20 / 45 w 46$ & P. columella & 61 \\
\hline & Careaçu & $22 \mathrm{~s} 02 / 45 \mathrm{w} 41$ & P. columella & 61 \\
\hline & Diamantina & $18 \mathrm{~s} 13 / 43 w 35$ & P. columella & 4 \\
\hline & Ferros & $19 \mathrm{~s} 12 / 43 \mathrm{w} 01$ & P. columella & 4 \\
\hline & Igarapé & $20 \mathrm{~s} 04 / 44 w 18$ & P. columella & 61 \\
\hline & Itabirito & $20 \mathrm{~s} 14 / 43 w 47$ & P. columella & 61 \\
\hline & Itajubá & $22 \mathrm{~s} 25 / 45 \mathrm{w} 28$ & P. columella ${ }^{+}$ & $\begin{array}{l}9 ; 61 ; 62 ; 24 ; 8 \\
\text { Fiocruz-CMM }\end{array}$ \\
\hline & Jaboticatubas & $19 \mathrm{~s} 30 / 43 \mathrm{w} 44$ & P. columella & 8; 63; Fiocruz-CMM \\
\hline & Januária & $15 \mathrm{~s} 26 / 44 w 21$ & P. columella & 64 \\
\hline & Juiz de Fora & $21 \mathrm{~s} 45 / 43 \mathrm{w} 20$ & P. columella & $\begin{array}{l}4 ; 65 ; 61 ; 83 \\
\text { Fiocruz-CMM }\end{array}$ \\
\hline & Lagoa Santa & $19 \mathrm{~s} 37 / 43 \mathrm{w} 53$ & P. columella & 59 \\
\hline & Machado & $21 \mathrm{~s} 39 / 45 w 55$ & P. columella & Fiocruz-CMM \\
\hline & Mariana & $20 s 21 / 43 w 25$ & P. columella & 66 \\
\hline & Nova Lima & $19 \mathrm{~s} 58 / 43 \mathrm{w} 50$ & P. columella & 59 \\
\hline & Ouro Fino & $22 \mathrm{~s} 16 / 46 \mathrm{w} 22$ & P. columella & 4 \\
\hline & Passos & $20 s 42 / 46 w 36$ & P. columella & MZUSP \\
\hline & Pedro Leopoldo & $19 \mathrm{~s} 36 / 44 \mathrm{w} 02$ & P. columella & 59 \\
\hline & Perdões & $21 \mathrm{~s} 09 / 45 w 08$ & P. columella & Fiocruz-CMM \\
\hline & Piranguinho & $22 \mathrm{~s} 23 / 45 \mathrm{w} 32$ & P. columella & 61 \\
\hline & Raposos & $19 \mathrm{~s} 57 / 43 w 48$ & P. columella & Fiocruz-CMM \\
\hline
\end{tabular}




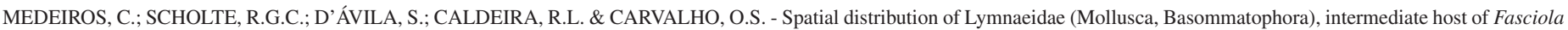
hepatica Linnaeus, 1758 (Trematoda, Digenea) in Brazil. Rev. Inst. Med. Trop. Sao Paulo, 56(3): 235-52, 2014.

Table 1

Distribution of Pseudosuccinea columella per municipalities and regions of Brazil (cont.)

\begin{tabular}{|c|c|c|c|c|}
\hline Regions/States & Localities & Geographic coordinates & Species & $\begin{array}{l}\text { Articles/“SpeciesLink" } \\
\text { (CRIA) network }\end{array}$ \\
\hline \multirow[t]{14}{*}{ Minas Gerais } & Ribeirão das Neves & 19s45/44w04 & P. columella & 59 \\
\hline & Rio Acima & $20 \mathrm{~s} 03 / 43 \mathrm{w} 47$ & P. columella & Fiocruz-CMM \\
\hline & Rio Doce & 20 s14/42w53 & P. columella & 8 \\
\hline & Sabinópolis & $18 \mathrm{~s} 39 / 43 w 04$ & P. columella & 4 \\
\hline & Santa Luzia & $19 \mathrm{~s} 44 / 43 w 53$ & P. columella & 59 \\
\hline & Santa Rita do Sapucaí & $22 \mathrm{~s} 14 / 45 w 43$ & P. columella & 61 \\
\hline & São Gonçalo do Sapucaí & $22 s 19 / 46 w 19$ & P. columella & $11 ; 61$ \\
\hline & Sete Lagoas & $19 \mathrm{~s} 26 / 44 w 14$ & P. columella & 11 \\
\hline & Timóteo & $19 \mathrm{~s} 34 / 42 w 38$ & P. columella & Fiocruz-CMM \\
\hline & Três Pontas & $21 \mathrm{~s} 21 / 45 w 30$ & P. columella & 24; Fiocruz-CMM \\
\hline & Ubá & $21 \mathrm{~s} 07 / 42 \mathrm{w} 56$ & P. columella & 4 \\
\hline & Varginha & $21 \mathrm{~s} 33 / 45 w 26$ & P. columella & 61 \\
\hline & Vespasiano & $19 s 41 / 43 w 55$ & P. columella & 59 \\
\hline & Viçosa & $20 \mathrm{~s} 45 / 42 w 52$ & P. columella & $11 ; 4$ \\
\hline \multirow[t]{16}{*}{ Espírito Santo } & Alegre & $20 s 45 / 41 w 31$ & P. columella & 68 \\
\hline & Atílio Vivacqua & $20 \mathrm{~s} 53 / 41 w 11$ & P. columella & 68 \\
\hline & Cachoeiro do Itapemirim & $20 \mathrm{~s} 49 / 41 w 06$ & P. columella & 68 \\
\hline & Castelo & $20 s 36 / 41 w 12$ & P. columella & 68 \\
\hline & Guaçuí & $20 \mathrm{~s} 45 / 41 w 40$ & P. columella & 68 \\
\hline & Guarapari & 20s65/40w51 & P. columella & UFES-Malacologia \\
\hline & Jerônimo Monteiro & $20 \mathrm{~s} 47 / 41 w 23$ & P. columella & 68 \\
\hline & Marataízes & $21 \mathrm{~s} 00 / 40 w 49$ & P. columella & 68 \\
\hline & Mimoso do Sul & $21 \mathrm{~s} 03 / 41 \mathrm{w} 22$ & P. columella & 68 \\
\hline & Muniz Freire & $20 \mathrm{~s} 27 / 41 \mathrm{w} 24$ & P. columella & 68 \\
\hline & Muqui & $20 \mathrm{~s} 56 / 41 w 20$ & P. columella & 68 \\
\hline & Piúma & $20 s 49 / 40 w 43$ & P. columella & 68 \\
\hline & Presidente Kennedy & 21s04/41w01 & P. columella & 68 \\
\hline & Serra & $20 \mathrm{~s} 12 / 40 \mathrm{w} 30$ & P. columella & UFES-Malacologia \\
\hline & Vartem Alta & $20 \mathrm{~s} 40 / 41 w 00$ & P. columella & 68 \\
\hline & Itapemirim & $21 \mathrm{~s} 00 / 40 w 49$ & P. columella & 68 \\
\hline \multicolumn{5}{|l|}{ 3) Midwest } \\
\hline \multirow[t]{12}{*}{ Goiás } & Barro Alto & $14 \mathrm{~s} 58 / 48 w 55$ & P. columella & 69 \\
\hline & Brasília & $15 \mathrm{~s} 46 / 47 \mathrm{w} 55$ & P. columella & 4 \\
\hline & Campinaçu & $13 \mathrm{~s} 44 / 48 w 34$ & P. columella & 69 \\
\hline & Campinorte & $14 \mathrm{~s} 18 / 49 w 88$ & P. columella & 69 \\
\hline & Colinas do Sul & $14 \mathrm{~s} 08 / 48 w 04$ & P. columella & 69 \\
\hline & Formosa & $15 \mathrm{~s} 30 / 47 w 20$ & P. columella & 4 \\
\hline & Goiânia & $16 s 41 / 49 w 13$ & P. columella & $\begin{array}{c}4 ; 24 ; 77 \\
\text { Fiocruz-CMM }\end{array}$ \\
\hline & Minaçu & $13 \mathrm{~s} 31 / 48 w 13$ & P. columella & 69 \\
\hline & Mineiros & $17 \mathrm{~s} 30 / 52 w 32$ & P. columella & 70 \\
\hline & Niquelândia & 14 s26/48w27 & P. columella & 69 \\
\hline & Santa Rita do Novo Destino & $15 \mathrm{~s} 06 / 49 \mathrm{w} 06$ & P. columella & 69 \\
\hline & Uruaçu & $14 \mathrm{~s} 29 / 49 w 07$ & P. columella & 69 \\
\hline
\end{tabular}


MEDEIROS, C.; SCHOLTE, R.G.C.; D'ÁVILA, S.; CALDEIRA, R.L. \& CARVALHO, O.S. - Spatial distribution of Lymnaeidae (Mollusca, Basommatophora), intermediate host of Fasciola hepatica Linnaeus, 1758 (Trematoda, Digenea) in Brazil. Rev. Inst. Med. Trop. Sao Paulo, 56(3): 235-52, 2014.

Table 1

Distribution of Pseudosuccinea columella per municipalities and regions of Brazil (cont.)

\begin{tabular}{|c|c|c|c|c|}
\hline Regions/States & Localities & Geographic coordinates & Species & $\begin{array}{c}\text { Articles/“SpeciesLink" } \\
\text { (CRIA) network }\end{array}$ \\
\hline Mato Grosso & Cuiabá & $15 \mathrm{~s} 35 / 56 w 05$ & P. columella & 4 \\
\hline \multirow[t]{2}{*}{ Mato Grosso do Sul } & Aquidauana & $20 \mathrm{~s} 27 / 55 w 46$ & P. columella & 4 \\
\hline & Bela Vista & $22 \mathrm{~s} 04 / 56 w 31$ & P. columella & 4 \\
\hline \multicolumn{5}{|l|}{ 4) Northeast } \\
\hline Bahia & Salvador & $12 \mathrm{~s} 58 / 38 \mathrm{w} 32$ & P. columella & 71 \\
\hline Ceará & Fortaleza & $3 \mathrm{~s} 45 / 38 w 32$ & P. columella & 8; Fiocruz-CMM \\
\hline Paraíba & Campina Grande & $7 \mathrm{~s} 12 / 35 \mathrm{w} 53$ & P. columella & $72 ; 73$ \\
\hline \multicolumn{5}{|l|}{ 5) North } \\
\hline \multirow[t]{6}{*}{ Amazonas } & Benjamin Constant & 4s22/70w01 & P. columella & 74 \\
\hline & Careiro & 3s81/60w34 & P. columella & Fiocruz-CMM \\
\hline & Coari & $4 \mathrm{~s} 05 / 63 \mathrm{w} 08$ & P. columella & Fiocruz-CMM \\
\hline & Manaus & $3 \mathrm{~s} 06 / 60 \mathrm{w} 03$ & P. columella & 74 \\
\hline & Tefé & $3 \mathrm{~s} 18 / 64 \mathrm{w} 42$ & P. columella & 71; Fiocruz-CMM \\
\hline & Iranduba & $3 \mathrm{~s} 28 / 60 w 18$ & P. columella & INPA-Mollusca \\
\hline Acre & Rio Branco & $9 \mathrm{~s} 58 / 67 w 48$ & P. columella & 24; Fiocruz-CMM \\
\hline
\end{tabular}

Table 2

Distribution of Galba viatrix, G. rupestris, G. cubensis, Lymnaea truncatula and Lymnaea sp. per municipalities and regions of Brazil

\begin{tabular}{|c|c|c|c|c|}
\hline Regions/States & Localities & Geographic coordinates & Species & $\begin{array}{c}\text { Articles/“SpeciesLink" } \\
\text { (CRIA) network }\end{array}$ \\
\hline \multicolumn{5}{|l|}{ 1) South } \\
\hline \multirow[t]{18}{*}{ Rio Grande do Sul } & Bagé & 31s19/54w06 & G. viatrix & 3 \\
\hline & Bom Jesus & $28 \mathrm{~s} 66 / 50 \mathrm{w} 43$ & Lymnaea sp. & MCP-Moluscos \\
\hline & Cambará do Sul & $28 s 97 / 50 w 31$ & Lymnaea sp. & MCP-Moluscos \\
\hline & Dom Pedrito & $30 \mathrm{~s} 58 / 54 w 40$ & G. viatrix $x^{+}$ & $4 ; 3 ; 7 ; 82$ \\
\hline & Erval Grande & $27 \mathrm{~s} 38 / 52 \mathrm{w} 57$ & Lymnaea sp. & MCP-Moluscos \\
\hline & Estrela & $28 \mathrm{~s} 86 / 51 \mathrm{w} 17$ & Lymnaea sp. & MCP-Moluscos \\
\hline & Ibirubá & $28 \mathrm{~s} 54 / 53 w 09$ & Lymnaea sp. & MCP-Moluscos \\
\hline & Jaguarão & $32 \mathrm{~s} 33 / 53 w 22$ & G. viatrix $x^{+}$ & $4 ; 3$ \\
\hline & Lavras do Sul & $30 \mathrm{~s} 48 / 53 \mathrm{w} 53$ & G. viatrix & 3 \\
\hline & Pelotas & $31 \mathrm{~s} 46 / 52 w 20$ & G. viatrix & 10 \\
\hline & Porto Alegre & $30 \mathrm{~s} 01 / 51 \mathrm{w} 13$ & Lymnaea sp. & MCP-Moluscos \\
\hline & Santa Vitória do Palmar & $33 \mathrm{~s} 31 / 53 w 22$ & G. viatrix $x^{+}$ & $\begin{array}{c}\text { 4; 14; } 3 ; 89 \\
\text { Fiocruz-CMM }\end{array}$ \\
\hline & São Borja & $28 \mathrm{~s} 39 / 55 w 59$ & Lymnaea sp. & MCP-Moluscos \\
\hline & São Francisco de Paula & $29 \mathrm{~s} 26 / 50 w 35$ & Lymnaea sp. & MCP-Moluscos \\
\hline & Selbach & $28 s 37 / 52 w 56$ & Lymnaea sp. & MCP-Moluscos \\
\hline & Três Coroas & $29 \mathrm{~s} 30 / 50 w 46$ & Lymnaea sp. & MCP-Moluscos \\
\hline & Vacaria & $28 \mathrm{~s} 48 / 50 \mathrm{w} 92$ & Lymnaea sp. & MCP-Moluscos \\
\hline & Viamão & 30s04/51w01 & Lymnaea sp. & MCP-Moluscos \\
\hline \multirow[t]{2}{*}{ Santa Catarina } & Campos Novos & $27 \mathrm{~s} 40 / 51 \mathrm{w} 22$ & Lymnaea sp. & MCP-Moluscos \\
\hline & Florianópolis & $27 \mathrm{~s} 35 / 48 w 33$ & G. viatrix & 21 \\
\hline
\end{tabular}




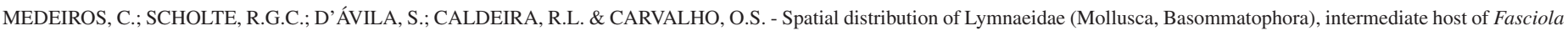
hepatica Linnaeus, 1758 (Trematoda, Digenea) in Brazil. Rev. Inst. Med. Trop. Sao Paulo, 56(3): 235-52, 2014.

Table 2

Distribution of G. viatrix, G. rupestris, G. cubensis and L. truncatula and Lymnaea sp. per municipalities and regions of Brazil (cont.)

\begin{tabular}{|c|c|c|c|c|}
\hline Regions/States & Localities & Geographic coordinates & Species & $\begin{array}{c}\text { Articles/“SpeciesLink” } \\
\text { (CRIA) network }\end{array}$ \\
\hline \multirow[t]{2}{*}{ Santa Catarina } & Seara & $27 \mathrm{~s} 12 / 52 \mathrm{w} 19$ & G. viatrix & $22 ; 4$ \\
\hline & & & L. rupestris & 23 \\
\hline Paraná & Curitiba & $25 \mathrm{~s} 25 / 49 \mathrm{w} 16$ & Lymnaea sp. & MZUSP \\
\hline \multicolumn{5}{|l|}{ 2) Southeast } \\
\hline \multirow[t]{4}{*}{ São Paulo } & Piracicaba & $22 \mathrm{~s} 43 / 47 \mathrm{w} 39$ & Lymnaea sp. & MZUSP \\
\hline & Presidente Prudente & $22 \mathrm{~s} 07 / 51 w 23$ & Lymnaea sp. & 40 \\
\hline & São Paulo & $23 s 32 / 46 w 38$ & Lymnaea sp. & MZUSP \\
\hline & Ubatuba & $23 \mathrm{~s} 25 / 45 \mathrm{w} 04$ & Lymnaea sp. & MZUSP \\
\hline \multirow[t]{11}{*}{ Rio de Janeiro } & Nova Friburgo & $22 \mathrm{~s} 15 / 42 \mathrm{w} 31$ & Lymnaea sp. & 45 \\
\hline & & & G. truncatula & Fiocruz-CMM \\
\hline & Paraíba do Sul & $22 \mathrm{~s} 08 / 43 \mathrm{w} 16$ & G. cubensis & 51 \\
\hline & Petrópolis & $22 \mathrm{~s} 21 / 43 w 08$ & G. cubensis & 51 \\
\hline & Rio de Janeiro & $22 \mathrm{~s} 53 / 43 \mathrm{w} 12$ & G. cubensis & 55 \\
\hline & & & Lymnaea sp. & MZUSP \\
\hline & Teresópolis & $22 \mathrm{~s} 23 / 42 \mathrm{w} 58$ & Lymnaea sp. & 50 \\
\hline & & & G. cubensis & 51 \\
\hline & & & G. truncatula & Fiocruz-CMM \\
\hline & Três Rios & $22 \mathrm{~s} 05 / 43 \mathrm{w} 12$ & G. cubensis & $51 ; 90$ \\
\hline & Vassouras & $22 \mathrm{~s} 40 / 43 w 66$ & Lymnaea sp. & 57 \\
\hline \multirow[t]{6}{*}{ Minas Gerais } & Belo Horizonte & $19 \mathrm{~s} 55 / 43 \mathrm{w} 56$ & G. cubensis & 60; Fiocruz-CMM \\
\hline & & & G. viatrix & 4 \\
\hline & Careaçu & $22 \mathrm{~s} 02 / 45 \mathrm{w} 41$ & Lymnaea sp. $^{+}$ & 79 \\
\hline & Ouro Branco & $20 \mathrm{~s} 30 / 43 \mathrm{w} 41$ & Lymnaea sp. & 67 \\
\hline & Rio Acima & $20 \mathrm{~s} 03 / 43 w 47$ & G. viatrix & 63 \\
\hline & & & G. truncatula & 60; Fiocruz-CMM \\
\hline
\end{tabular}

3) North

\begin{tabular}{lccc}
\hline Pará & Belém & Lymnaea sp. & MZUSP \\
\hline Legends for the tables 1 and 2: The numbers at the column Articles of the tables 1 and 2 are references to the authors that record the occurrence of specimens of Lymnaei-
\end{tabular} dae. 1: Simões (2002); 2: Amaral et al. (2007); 3: Ueno et al. (1982); 4: Paraense (1982a); 5: Mattos et al. (1997); 6: Pereira et al. (2000a); 7: Mattos \& Ueno (1985); 8: Coelho (2007); 9: Coelho et al. (2009); 10: Rey (1957); 11: Dacal et al. (1988); 12: Gonzales et al. (1974); 13: Indrusiak (1983); 14: Müller et al. (1998); 15: Martello et al. (2008); 16: Pereira et al. (2000b); 17: Agudo (2004) apud Agudo-Padrón (2008); 18: Agudo (2005); 19: Agudo (2007); 20: Agudo (2006); 21: Agudo-Padrón (2008); 22: Hubendick (1951); 23: Paraense (1982b); 24: Cardoso et al. (2006); 25: Bellato et al. (1996); 26: Queiroz et al. (2002); 27: Amaral \& Busetti (1979) apud Buseti (1982); 28: Busetti (1982); 29: Vaz et al. (1987); 30: Vaz et al. (1986); 31: Vaz et al. (1983); 32: Muniz (2007); 33: Gouveia \& Henry (1990); 34: Ueta (1976); 35: Magalhães et al. (1991); 36: Carvalho et al. (2001); 37: Vaz et al. (1992); 38: Maure et al. (1998); 39: Ueta (1980); 40: Tostes et al. (2004); 41: Oliveira et al. (2002); 42: Thiengo et al. (2004a); 43: Thiengo et al. (2006); 44: Thiengo et al. (2002b); 45: Thiengo et al. (2002a); 46: Thiengo et al. (2001); 47: Gomes et al. (2002); 48: Fiuza et al. (2006); 49: Thiengo et al. (2004b); 50: Thiengo et al. (1998); 51: Rezende et al. (1973); 52: Andrade Neto et al. (1999); 53: Medeiros et al. (2002); 54: Fernandez et al. (2001); 55: Nuernberg (1978); 56: Pinheiro \& Amato (1996); 57: Lutz (1921); 58: Souza et al. (2002); 59: Souza et al. (1998); 60: Cardoso (2004); 61: Lima et al. (2009); 62: Coelho \& Lima (2003); 63: Carvalho et al. (2004); 64: Vianna et al. (2007); 65: Martins \& Alves (2008); 66: Souza et al. (2006); 67: Silva et al. (1994); 68: Almeida (2010); 69: Thiengo et al. (2005); 70: Teles et al. (1991); 71: Paraense (1986); 72: Abílio \& Watanabe (1998); 73: Abílio et al. (2006); 74: Paraense (1983); 75: Amato et al. (1986); 76: Bruno et al. (1995); 77: Araújo et al. (1995); 78: Silva (2010); 79: Oliveira (2008); 80: Ueta (1977); 81: Silva-Santos et al. (1994); 82: Silva-Santos et al. (1987); 83: Martins \& Alves (2010); 84: Veitenheimer-Mendes (1992); 85: Pile et al. (1999); 86: Braun (2005); 87: Morretes (1949); 88: Pinheiro et al. (2009); 89: Lara et al. 1988; 90: Nuernberg et al. 1983; 91: Luz et al. (1994). Fiocruz-CMM represents data obtained from the Collection of Medical Malacology, CPqRR, Fiocruz/MG. MCP-Moluscos represents data obtained from the Collection of Molluscs of Museum of Science and Technology, PUCRS. INPAMollusca represents data obtained from the Collection of Molluscs of National Institute of Research of Amazônia, INPA. UFES-Malacologia represents data obtained from the Malacological Collection of Biological Science Department, DCBio/UFES. MZUSP represents data obtained from the Malacological Collection of Zoology Museum of University of São Paulo. The symbol ${ }^{+}$represents snails found naturally infected with Fasciola hepatica. 
MEDEIROS, C.; SCHOLTE, R.G.C.; D’ÁVILA, S.; CALDEIRA, R.L. \& CARVALHO, O.S. - Spatial distribution of Lymnaeidae (Mollusca, Basommatophora), intermediate host of Fasciola hepatica Linnaeus, 1758 (Trematoda, Digenea) in Brazil. Rev. Inst. Med. Trop. Sao Paulo, 56(3): 235-52, 2014.

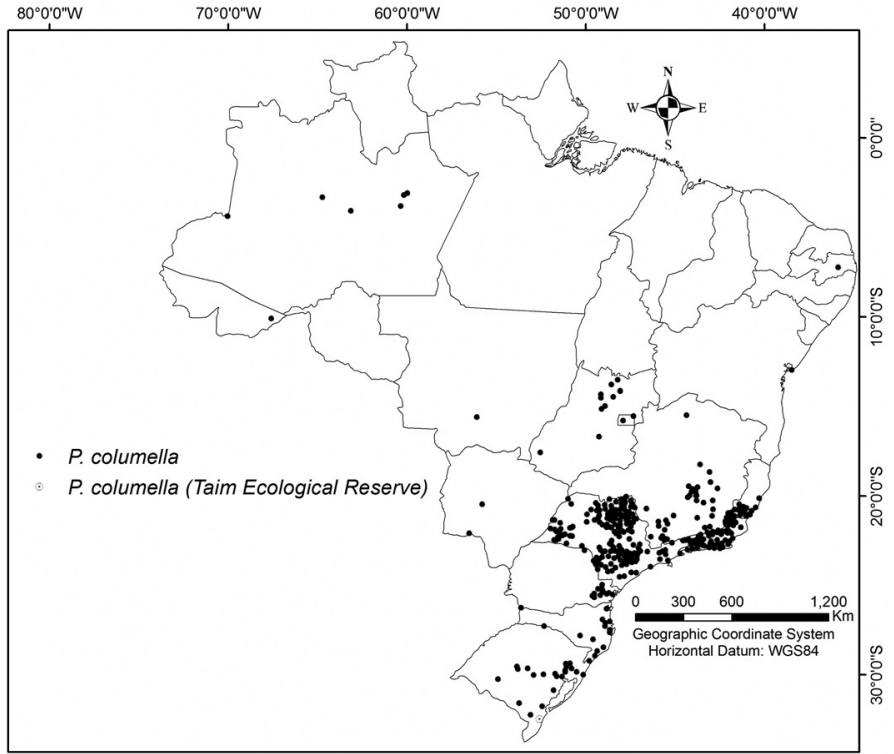

Fig. 1 - Spatial distribution of Pseudosuccinea columella in Brazil.

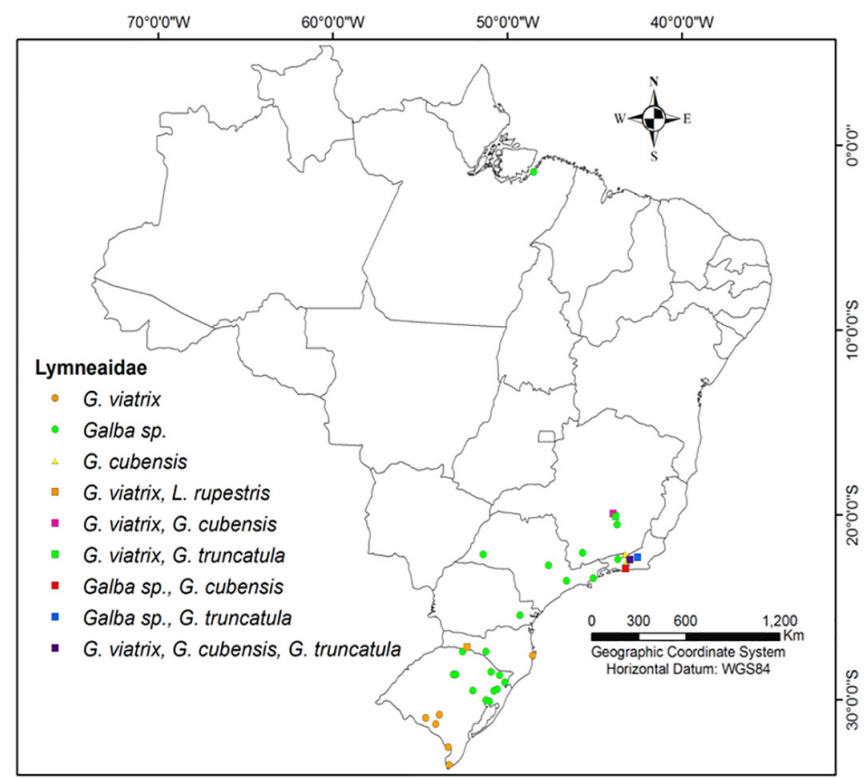

Fig. 2 - Spatial distribution of Galba viatrix, G. cubensis, G. truncatula, Lymnaea rupestris, and Lymnaea sp. in Brazil.

In relation to the others susceptible species, G. cubensis occurs only in the Southeast, mainly in Rio de Janeiro State ${ }^{19,60,76}$. There was a gap of 30 years in its record and until now its presence in other regions was not reported. Galba truncatula is also susceptible to $F$. hepatica, however with few records $(0.7 \%)$ it has never been found naturally infected in Brazil. Lymnaea rupestris was only described and found in one locality and there is no information about its susceptibility. Due to these facts, the role in the transmission of fasciolosis of the species above mentioned remains unknown.

In the present study, it was possible to observe the occurrence of

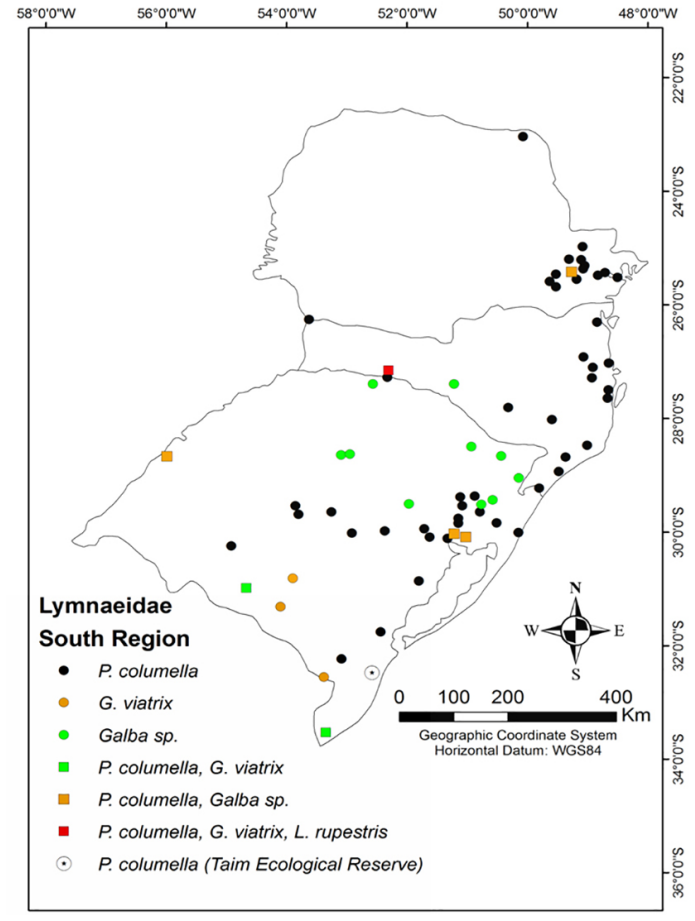

Fig. 3 - Spatial distribution of Lymnaeidae in the South region of Brazil.

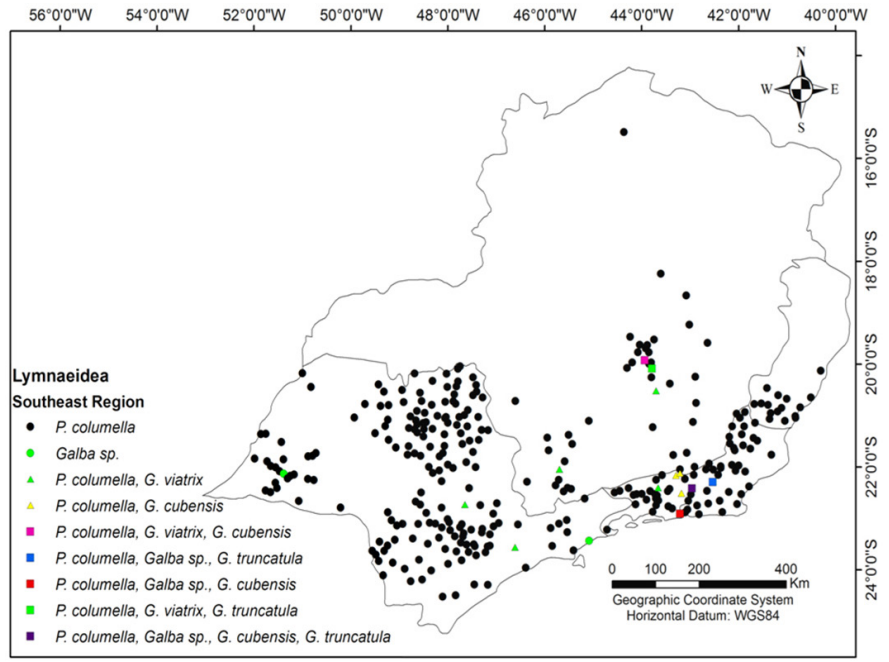

Fig. 4 - Spatial distribution of Lymnaeidae in the Southeast region of Brazil.

P. columella in several localities of the South (four municipalities) and Southeast (seven municipalities) and of G. viatrix in the South (three municipalities) naturally infected by $F$. hepatica (Table 1 and 2). In the South region, the presence of extensive endemic areas of fasciolosis is frequent, while in the Southeast and Midwest regions there are only small endemic areas ${ }^{41}$. Some environmental and climatic factors in the South region provide favorable habitats for the intermediate host snails of $F$. hepatica, such as low elevation (where there are numerous rice fields), clay soil, humid areas, and a temperate climate. These habitats, as shown by UENO et al. ${ }^{98}$, contribute to the maintenance of the snails' life cycle in this region. 


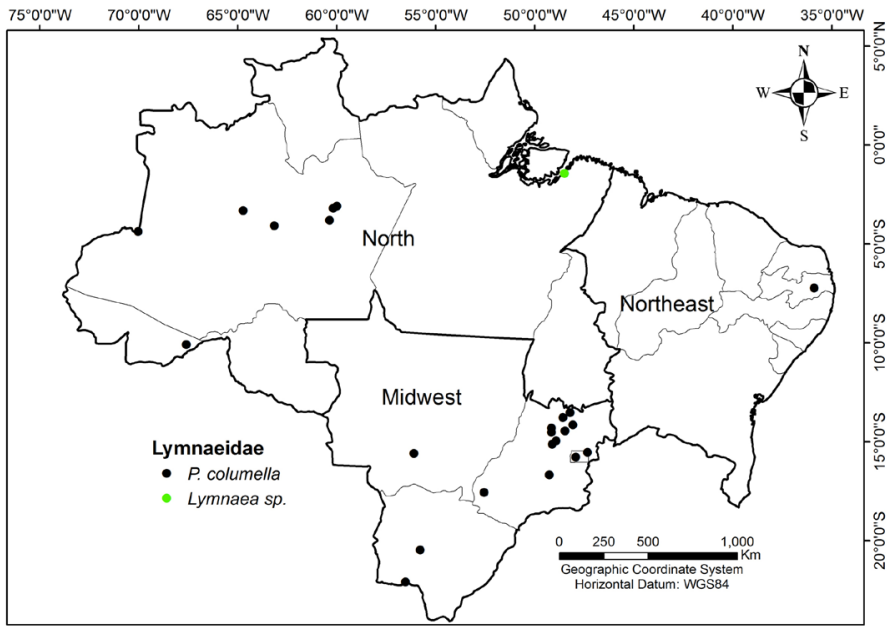

Fig. 5 - Spatial distribution of Lymnaeidae in the Midwest, North and Northeast regions of Brazil.

Despite the great efforts made by research groups, some important issues related to the nature and precision of the presence of lymnaeid species in Brazil need to be considered when interpreting our findings. The complete distribution of intermediate host snails of $F$. hepatica in Brazil remains unknown, especially due to the large areas that need to be surveyed mainly in the North and Midwest regions. Other restraining factors include the insufficient number of researchers involved in the taxonomy area, which may cause difficulties in identifying the snails at a species level due to the confusing taxonomic situation of the Lymnaeidae family. It was confirmed by our study that in several localities (25 municipalities; $6 \%$ ) the specific identification was not possible to be done. The identification of specimens is important since knowing the species that act as intermediate hosts in a particular area, we can define priority strategies regarding intermediate hosts biology to control fasciolosis. In Careaçu, MG, the occurrence of naturally-infected specimens without specific identification was reported (Table 2).

Another important point concerns the validity of the species identification used from the literature data and malacological collections due to systematics confusion of lymnaeids snails. This identification is essential in order to justify the distribution of lymnaeid species in Brazil. Regarding L. rupestris and P. columella, there is no problem because these two species can be easily identified using a set of reliable morphological characters and the literature data can be used with a reasonable confidence. However, this is not the case regarding the three other remaining species, G. viatrix, G. cubensis and G. truncatula, because these small species cannot be separated morphologically and only molecular markers allow a clear separation ${ }^{14,27,28,56,73,77}$. In our literature search we identified only 19 localities where the specific identification might be a concern (10 localities with $G$. viatrix, six localities with $G$. cubensis and three localities with $G$. truncatula). Only in the occurrence of $G$. cubensis for Belo Horizonte, $\mathrm{MG}^{19}$, G. truncatula for Rio Acima, $\mathrm{MG}^{22}$ and the data from Fiocruz-CMM have the authors already carried out molecular identification.

Studies on the lymnaeid fauna should be increased all over Brazil, mainly where the information is scarce or nonexistent, with the objective to learn more about the spatial distribution of the intermediate host snail of $F$. hepatica. The methodology used and the intermediate host snail final maps could be useful in identifying priority areas for control interventions of fasciolosis, so that limited resources could be allocated most effectively.

\section{RESUMO}

\section{Distribuição espacial de Lymnaeidae (Mollusca, Basommatophora), hospedeiros intermediários de Fasciola hepatica Linnaeus, 1758 (Trematoda, Digenea) no Brasil}

Moluscos da família Lymnaeidae são hospedeiros intermediários no ciclo biológico de Fasciola hepatica, agente etiológico da fasciolose, doença parasitária de importância médica para humanos e animais. O presente trabalho teve como objetivo datar e mapear a distribuição espacial dos hospedeiros intermediários de F. hepatica no Brasil. Os dados de distribuição das espécies de limneídeos foram obtidos das Coleções de Malacologia Medica (Fiocruz-CMM, CPqRR) e de Malacologia do Museu de Zoologia da Universidade de São Paulo (MZUSP), rede "SpeciesLink" (CRIA) e através de pesquisas sistemáticas na literatura. Os mapas de distribuição dos limneideos mostram que Pseudosuccinea columella é a espécie mais comum e distribuída nas regiões Sul e Sudeste com poucos registros nas regiões Centro-Oeste, Norte e Nordeste. Além disso, na região Sul os registros para G. viatrix são escassos e existe apenas um único relato de ocorrência para $L$. rupestris. Os dados obtidos resultaram no primeiro mapa de distribuição espacial das espécies de Lymnaeidae no Brasil, informação básica e relevante para estruturação de políticas de controle da fasciolose humana e animal.

\section{ACKNOWLEDGEMENTS}

We would like to thank the Health Sciences Library "Prof. Zigman Brener" of the Research Center René Rachou and the assistant Nuzia Pereira dos Santos for allowing access to scientific information through the availability of the required articles. We thank Dr. Luiz Ricardo Simone, Zoology Museum of University of São Paulo for allowing access to the data from the Malacological Collection of Zoology Museum of University of São Paulo. We thank Dr. Jean Pierre Pointier, University of Perpignan, France, for their important suggestions on the manuscript. We also thank José Geraldo Amorim, Laboratory of Medical Helminthology and Malacology, CPqRR, Fiocruz/MG for collecting snails of the Collection Fiocruz-CMM. This work was partially sponsored by the Fapemig (Process APQ-01633-10), CNPq (Process 150386/2012-5), CPqRR - Programa de Excelência em Pesquisa, PROEP/CPqRR (Process 401968/2012-0) and CNPq - Doutorado Sanduiche no Exterior, SWE/ CNPq (Process 245926/2012-7).

\section{REFERENCES}

1. Abílio FJP, Watanabe T. Ocorrência de Lymnaea columella (Gastropoda: Lymnaeidae), hospedeiro intermediário de Fasciola hepatica, para o estado da Paraíba, Brasil. Rev Saude Publica. 1998;32:184-5.

2. Abílio FJP, Fonseca-Gessner AA, Leite RM, Ruffo TLM. Gastrópodes e outros invertebrados do sedimento e associados à macrófita Eichhornia crassipes de um açude hipertrófico do semi-árido paraibano. Rev Biol Cien Terra. 2006;6:165-78.

3. Agudo AI. The freshwater mussels/naiads of Upper and Medium sessions of the Uruguay River Basin, Southern Brazil: a brief approach to its knowledge. Ellipsaria. 2005;7:9 10. 
MEDEIROS, C.; SCHOLTE, R.G.C.; D'ÁVILA, S.; CALDEIRA, R.L. \& CARVALHO, O.S. - Spatial distribution of Lymnaeidae (Mollusca, Basommatophora), intermediate host of Fasciola hepatica Linnaeus, 1758 (Trematoda, Digenea) in Brazil. Rev. Inst. Med. Trop. Sao Paulo, 56(3): 235-52, 2014.

4. Agudo AI. Intermediate host mollusks (Gastropoda: Pulmonata) of parasitic diseases in Santa Catarina's State, Southern Brazil, with inclusion of new records to add to regional inventory. Ellipsaria. 2006;8:11-3.

5. Agudo AI. Exotic isolated occurrence of the mussel naiad Leila blainvilleana (Lea, 1834) in a freshwater coastal lagoon of the Santa Catarina Island, Southern Brazil. Ellipsaria. 2007;9:10-2

6. Agudo-Padrón AI. Listagem sistemática dos moluscos continentais ocorrentes no Estado de Santa Catarina, Brasil. Comun Soc Malacol Urug. 2008;9:147-79.

7. Almeida BR. Malacologia dos gêneros Lymnaea e Biomphalaria na Mesorregião Sul Espírito-Santense, e a avaliação de extratos de Melia azedarach, Azadirachta indica, e Cymbopogon winterianus como agentes moluscicidas [dissertação]. Alegre: Universidade Federal do Espírito Santo; 2010.

8. Amaral HLC, Pepe MS, Müller G, Rodrigues AP, Berne MEA. Suscetibilidade de populações de Lymnaea columella à infecção por Fasciola hepatica. IX Encontro de Pós-Graduação; XVI Congresso de Iniciação Científica. Available from: http:// www.ufpel.edu.br/cic/2007/cd/pdf/CB/CB_01122.pdf; 2007

9. Amato SB, Rezende HEB, Gomes DC, Freire NMSR. Epidemiology of Fasciola hepatica infection in the Paraiba River Valley, São Paulo, Brasil. Vet Parasitol. 1986;22:275-84.

10. Andrade Neto JL, Carneiro Filho M, Luz E, Siciliano RF, Oliveira Filho AG, Pisani JC. Human fascioliasis in The Metropolitan Area of Curitiba, Brazil - Evaluation of the foci of infection and report of nine cases treated with triclabendazole. Braz J Infect Dis. 1999;3:220-5.

11. Araújo JLB, Linhares GFC, Paçô JM. Ocorrência de Lymnaea columella (Say , 1817) (Mollusca, Pulmonata, Lymnaeidae), no Estado de Goiás. Considerações sobre os parasitos por ela transmitidos. Rev Patol Trop. 1995;24:291-300.

12. Araújo JLB, Linhares GFC, Oliveira APM, Amoril JG, Freitas MR, Costa IC, et al. Infecções autóctones de bovinos por Fasciola hepatica Linnaeus, 1758 (Trematoda, Fasciolidae) no estado de Goiás, Brasil. Rev Patol Trop. 2007;36:96-100.

13. Baker FC. The Natural History Survey of the Chicago Academy of Sciences. Chicago. Proccedings of the American Association of Museums; 1908;2:15-19.

14. Bargues MD, Artigas P, Mera y Sierra RL, Pointier JP, Mas-Coma S. Characterisation of Lymnaea cubensis, L. viatrix and L. neotropica n. sp., the main vectors of Fasciola hepatica in Latin America, by analysis of their ribosomal and mitochondrial DNA. Ann Trop Med Parasitol. 2007;101:621-41.

15. Bellato V, Souza AP, Sobrinho DAP. Prevalência de Fasciola hepatica em bovinos do município de Urubicí - SC. Florianópolis: Universidade \& Desenvolvimento; 1996. (Série Científica). v. 3. p. 38-48.

16. Braun BS. Comunidades de molusco em córregos impactados da macrobacia das lagoas costeiras de Jacarepaguá, RJ. [dissertação]. Rio de Janeiro: Universidade Estadual do Rio de Janeiro; 2005

17. Bruno SF, Mattos DG, Silva EV, Francis M, Brito DB. Fasciola hepatica (Linnaeus 1758) em bovinos do município de Cachoeiras de Macacu. Estado do Rio de Janeiro, Brasil. Parasitol al día. 1995;19:65-8.

18. Busetti ET. Informações adicionais sobre a fasciolose hepática em Curitiba (Estado do Paraná, Brasil). Rev Inst Med Trop Sao Paulo. 1982;24:104-6.

19. Cardoso PCM. Diferenciação molecular de moluscos do gênero Lymnaea (Gastropoda, Lymnaeidae) utilizando a PCR-RFLP e o estudo da variabilidade genética de $L$. columella pela RAPD. [dissertação]. Belo Horizonte: Centro de Pesquisas Rene Rachou, Fundação Oswaldo Cruz; 2004.

20. Cardoso PCM, Caldeira RL, Lovato MB, Coelho PMZ, Berne MEA, Müller G, et al. Genetic variability of Brazilian populations of Lymnaea columella (Gastropoda: Lymnaeidae), an intermediate host of Fasciola hepatica (Trematoda: Digenea). Acta Trop. 2006;97:339-45.
21. Carvalho GA, Ueta MT, Andrade CFS. Búsqueda de xifidiocercarias (Trematoda) en moluscos de agua dulce recolectados en nueve municipios del Estado de São Paulo, Brasil. Bol Chil Parasitol. 2001;56:3-9.

22. Carvalho OS, Cardoso PCM, Lira PM, Rumi A, Roche A, Berne E, et al. The use of the polymerase chain reaction and restriction fragment length polymorphism technique associated with the classical morphology for characterization of Lymnaea columella, L. viatrix, and $L$. diaphana (Mollusca: Lymnaeidae). Mem Inst Oswaldo Cruz. 2004;99:503-7.

23. Carvalho OS, Passos LKJ, Mendonça CLFG, Cardoso PCM, Caldeira RL. Moluscos de importância médica no Brasil. Belo Horizonte: Fiocruz/Centro de Pesquisas Rene Rachou; 2008

24. Coelho LHL, Lima WS. Population dynamics of Lymnaea columella and its natura infection by Fasciola hepatica in the State of Minas Gerais, Brazil. J Helminthol. 2003;77:7-10.

25. Coelho LHL. Lymnaea columella: dinâmica de populações em Itajubá, MG suscetibilidade à infecção por Fasciola hepatica em associações simpátricas e alopátricas entre parasito e hospedeiro. [tese]. Belo Horizonte: Universidade Federal de Minas Gerais; 2007.

26. Coelho LHL, Lima WS, Guimarães MP. Sympatric and allopatric combinations of Lymnaea columella and Fasciola hepatica from southern and south-eastern Brazil. J Helminthol. 2009;83:285-8.

27. Correa AC, Escobar JS, Durand P, Renaud F, David P, Jarne P, et al. Bridging gaps in the molecular phylogeny of the Lymnaeidae (Gastropoda: Pulmonata), vectors of Fascioliasis. BMC Evol Biol. 2010;10:1-12.

28. Correa AC, Escobar JS, Noya O, Velásquez LE, González-Ramírez C, Hurtrez-Boussès $\mathrm{S}$, et al. Morphological and molecular characterization of Neotropic Lymnaeidae (Gastropoda: Lymnaeoidea), vectors of fasciolosis. Infect Genet Evol. 2011;11:197888 .

29. Dacal ARC, Costa HMD, Leite ACR. Susceptibilidade de Lymnaea (Pseudosuccinaea) columella (Say, 1817) exposta à infecção por miracídios de Fasciola hepatica (Linnaeus, 1758). Rev Inst Med Trop Sao Paulo. 1988;30:361-9.

30. Fernandez MA, Thiengo SC, Boaventura MF. Gastrópodes límnicos do Campus de Manguinhos, Fundação Oswaldo Cruz, Rio de Janeiro, RJ. Rev Soc Bras Med Trop. 2001;34:279-82

31. Fiuza VRS, Gomes FF, Paes RB, Oliveira FCR. Lymanea columella Say, 1817 (Gastropoda: Lymnaeidae): distribuição espacial no Município de Campos dos Goytacazes, RJ. Rev Univ Rural Rio de Janeiro (Série Ciências da Vida). 2006;26(Suppl):199-200.

32. Gomes FF, Oliveira FCR, Pile EA, Lopes CWG. Estabelecimento de foco de fasciolose hepática em propriedade do município de Campos dos Goytacazes no estado do Rio de Janeiro, Brasil. Rev Bras Parasitol Vet. 2002;11:53-6.

33. Gonzales JC, Sanchez VM, Thome JW, Gonçalves PC, Oliveira CMB. Lymnaea columella, hospedeiro intermediário de Fasciola hepatica L. 1758, no Rio Grande do Sul, Brasil. Arq Fac Vet Rio Grande do Sul. 1974;2:37-40.

34. Gouveia L, Henry R. Efeito do tamanho e temperatura no consumo de oxigênio de Lymnaea columella Say, 1817 (Mollusca, Gastropoda). Ci Cult. 1990;42:397-402.

35. Gutiérrez A, Perera G, Yong M, Lin W. The effect of isolation on the life-history traits of Pseudosuccinea columella (Pulmonata: Lymnaeidae). Mem Inst Oswaldo Cruz. 2001;96:577-81.

36. Honer MR. Aspectos da epidemiologia da fasciolose. In: $1^{\circ}$ Seminário Nacional sobre Parasitoses de Bovinos; 1979; Campo Grande. Anais. Brasília: EMBRAPA/CNPGC; 1979. p. 151-65

37. Hubendick B. Recent Lymnaeidae. Their variation, morphology, taxonomy, nomenclature and distribution. K Sven Vetenskapsakad Handl. 1951;3:1-223. 
MEDEIROS, C.; SCHOLTE, R.G.C.; D'ÁVILA, S.; CALDEIRA, R.L. \& CARVALHO, O.S. - Spatial distribution of Lymnaeidae (Mollusca, Basommatophora), intermediate host of Fasciola hepatica Linnaeus, 1758 (Trematoda, Digenea) in Brazil. Rev. Inst. Med. Trop. Sao Paulo, 56(3): 235-52, 2014.

38. Indrusiak LF. Inventory of the malacological fauna from Ibibuí Mirim River, RS. Ci Nat. 1983;5:127-34.

39. Lara SIM, Fernandez FG, Muller G, Silveira P. Biologia de Lymnaea viatrix Orb., 1835 II. Desenvolvimento, postura e longevidade. Arq Bras Med Vet Zootec. 1988;40:10113 .

40. Lessa CSS, Scherer PO, Vasconcelos MC, Freire LS, Santos JAA, Freire NMS. Registro de Fasciola hepatica em eqüinos (Equus caballus), caprinos (Capra kircus) e ovinos (Ovis aries) no município de Itaguaí, Rio de Janeiro, Brasil. Rev Bras Ci Vet. 2000;7:63-4

41. Lima WS, Soares LRM, Barçante TA, Guimarães MP, Barçante JMP. Occurrence of Fasciola hepatica (Linnaeus, 1758) infection in Brazilian cattle of Minas Gerais, Brazil. Rev Bras Parasitol Vet. 2009;18:27-30.

42. Lutz A. Sobre a ocorrência de Fasciola hepatica no estado do Rio de Janeiro. Bol Inst Oswaldo Cruz. 1921;1:9-13.

43. Luz E, Gazda CM, Yada RS. Fasciolose animal no Estado do Paraná: análise de dados. Arq Biol Tecnol. 1992;35:777-80.

44. Luz E, Vieira AM, Cesar TCP. Aspectos de Lymnaea columella Say, 1817, Physa cubensis Pfeiffer, 1839 e Physa marmorata Guilding, 1928 (Mollusca-Pulmonata) no primeiro planalto e litoral paranaense. Arq Biol Tecnol. 1994;37:667-71.

45. Magalhães LA, Zanotti-Magalhães EM, Carvalho JF, Faraone M. Atração miraxonal exercida por Biomphalaria straminea, Lymnaea columella e Physa sp., sobre miracídios de Schistosoma mansoni da linhagem BH. Rev Saude Publica. 1991;25:237.

46. Martello AR, Nunes IGW, Boelter RA, Leal LA. Malacofauna límnica associada a macrófitas aquáticas do rio Iguariaçá, São Borja, RS, Brasil. Ci Nat. 2008;30:27-41.

47. Martins RT, Alves RG. Occurrence of Naididae (Annelida: Oligochaeta) from three gastropod species in irrigation fields in southeastern Brazil. Biota Neotrop. 2008;8:255-7.

48. Martins RT, Alves RG. Occurrence of Chaetogaster limnaei K. von Baer, 1927 (Oligochaeta, Naididae) associated with Gastropoda mollusks in horticultural channels in Southeastern Brazil. Braz J Biol. 2010;70:1055-7.

49. Mas-Coma S, Angles R, Strauss W, Esteban JG, Oviedo JA, Buchon P. Human fascioliasis in Bolivia: a general analysis and a critical review of existing data. Res Rev Parasitol. 1995;55:73-9.

50. Mas-Coma S, Esteban JG, Bargues MD. Epidemiology of human fascioliasis: a review and proposed new classification. Bull World Health Organ. 1999;77:340-6.

51. Mattos MJT, Ueno H. Manutenção de Lymanea viatrix Orbigny, 1835 em condições laboratoriais. Hora Vet. 1985;5:48-50.

52. Mattos MJT, Ueno H. Suscetibilidade de Lymnaea viatrix e L. columella às infecções experimentais com Fasciola hepatica. Pesq Agropec Bras. 1989;24:615-22.

53. Mattos MJT, Ueno H, Gonçalves PC, Almeida JEM. Ocorrência estacional e bioecologia de Lymnaea columella Say, 1817 (Mollusca, Lymnaeidae) em habitat natural no Rio Grande do Sul. Rev Bras Med Vet. 1997;19:248-52.

54. Maure EAP, Bustamante M, Serra-Freire NM, Gomes DC. Dinâmica de Lymnaed columella (Say, 1817), hospedeiro intermediario de Fasciola hepatica (Linnaeus, 1758) em municípios do estado de São Paulo, Brasil. Braz J Vet Res Anim Sci. $1998 ; 35: 151-5$

55. Medeiros ADS, Cruz OJ, Fernandez MA. Esquistossomose mansônica e distribuição dos moluscos límnicos em criadouros naturais no Município de Niterói, Rio de Janeiro, Brasil. Cad Saude Publica. 2002;18:1463-8.
56. Meunier C, Tirard C, Hurtrez-Boussès S, Durand P, Bargues MD, Mas-Coma S, et al. Lack of molluscan host diversity and the transmission of an emerging parasitic disease in Bolivia. Mol Ecol. 2001;10:1333-40.

57. Morretes FL. Ensaio de catálogo dos moluscos do Brasil. VII. Curitiba. Arquivos do Museu Paranaense. 1949;7:1-216.

58. Müller G, Lara SIM, Silveira Jr P, Antunes PL. Acompanhamento laboratorial do ciclo biológico de Lymnaea viatrix, hospedeiro intermediário de Fasciola hepatica. Rev Bras Agroci. 1998;4:172-6.

59. Muniz C. Levantamento da malacofauna límnica e aspectos ecológicos de focos de esquistossomose em Ana Dias, Vale do Ribeira-SP. [dissertação]. São Paulo: Universidade de São Paulo; 2007.

60. Nuernberg S. Estudos experimentais com Lymnaea cubensis Pfeiffer, 1839 (Mollusca, Gastropoda, Basommatophora, Lymnaeaidae) como hospedeiro intermediário de Fasciola hepatica L. 1758 (Trematoda, Fasciolidae), no Estado do Rio de Janeiro. [tese]. Rio de Janeiro: Universidade Federal Rural do Rio de Janeiro; 1978.

61. Nuernberg S, Rezende HEB, Serra-Freire NM, Gomes PAC, Araújo JLB. Biologia e susceptibilidade de Lymnaea cubensis (Mollusca, Lymnaeidae) a infecções por Fasciola hepatica em condições experimentais. Pesq Vet Bras. 1983;3:1- 10.

62. Oliveira EL. Prevalência e fatores associados à distribuição da Fasciola hepatica Linnaeus, 1758 em bovinos dos municípios de Careaçú e Itajubá, região da bacia do rio Sapucaí - Minas Gerais. [dissertação]. Belo Horizonte: Universidade Federal de Minas Gerais; 2008.

63. Oliveira SM, Fuji TU, Sposito Filha E, Martins AMCRPF. Ocorrência de Lymnaea columella Say, 1817 infectada naturalmente por Fasciola hepatica (Linnaeus, 1758), no vale do Ribeira, São Paulo, Brasil. Arq Inst Biol (São Paulo). 2002;69:29-37.

64. Paraense WL. Lymnaea rupestris sp. n. from Southern Brazil (Pulmonata: Lymnaeidae) Mem Inst Oswaldo Cruz. 1982a;77:437-43.

65. Paraense WL. Lymnaea viatrix and Lymnaea columella in the Neotropical Region: a distributional outline. Mem Inst Oswaldo Cruz. 1982b;77:181-8.

66. Paraense WL. Lymnaea columella in Northern Brazil. Mem Inst Oswaldo Cruz $1983 ; 78: 477-82$

67. Paraense WL. Lymnaea columella: two new Brazilian localities in the states of Amazonas and Bahia. Mem Inst Oswaldo Cruz. 1986;8:121-3.

68. Pereira D, Konrad HG, Paloski NI. Gastrópodos límnicos da bacia do rio Camaquã, RS, Brasil. Acta Biologica Leopoldensia. 2000a;22:55-66.

69. Pereira D, Veitenheimer-Mendes IL, Mansur MCD, Silva MCP. Malacofauna límnica do sistema de irrigação do arroio Capivara, Triunfo, RS, Brasil. Biociências. $2000 b ; 8: 137-57$.

70. Pile E, Lessa CSS, Scherer PO, Santos JAA, Vasconcellos MC. Ocurrencia de fasciolosis bovina en Itaguaí, Río de Janeiro, Brasil. Parasitol al día. 1999;23:123-4.

71. Pinheiro J, Amato SB. Consumo de oxigênio por Lymnaea columella (Mollusca, Gastropoda) infectada com estágios larvais de Fasciola hepatica (Platyhelminthes, Digenea). Rev Univ Rural (Rio J) (Série Ciências da Vida). 1996;18:95-9.

72. Pinheiro J, Maldonado Junior A, Lanfredi RM. Physiological changes in Lymnaed columella (Say, 1817) (Mollusca, Gastropoda) in response to Echinostoma paraense Lie and Basch, 1967 (Trematoda: Echinostomatidae) infection. Parasitol Res. 2009;106:55-9.

73. Pointier JP, González-Salas C, Gutiérrez A, Arenas JA, Bargues MD, et al. Anatomica studies of sibling species within neotropical lymnaeids, snail intermediate hosts of fascioliasis. Mem Inst Oswaldo Cruz. 2006;101:431-5. 
MEDEIROS, C.; SCHOLTE, R.G.C.; D'ÁVILA, S.; CALDEIRA, R.L. \& CARVALHO, O.S. - Spatial distribution of Lymnaeidae (Mollusca, Basommatophora), intermediate host of Fasciola hepatica Linnaeus, 1758 (Trematoda, Digenea) in Brazil. Rev. Inst. Med. Trop. Sao Paulo, 56(3): 235-52, 2014.

74. Queiroz VS, Luz E, Leite LC, Círio SM. Fasciola hepatica (Trematoda, Fasciolidae): estudo epidemiológico nos municípios de Bocaiúva do Sul e Tunas do Paraná (Brasil). Acta Biol Parasitol. 2002;31:99-111.

75. Rey L. Fasciola hepatica no gado, no Rio Grande do Sul. Investigações sobre a possibilidade de ocorrência de casos humanos. Rev Bras Malar. 1957;9:473-83.

76. Rezende HEB, Araujo JLB, Gomes PAC, Nuernberg S, Neto MP, Oliveira GP et al. Notas sobre duas espécies de Lymnaea Lamark, 1799, hospedeiros intermediários da Fasciola hepatica no estado do Rio de Janeiro. (Mollusca, Gastropoda, Basommatophora, Lymnaeidae). Arq Univ Fed Rural. 1973;3:21-3.

77. Samadi S, Roumégoux A, Bargues MD, Mas-Coma S, Yong M, Pontier JP. Morphological studies of Lymnaeidae snails from human fascioliasis endemic zone of Bolivia. J Molluscan Stud. 2000;66:31-44.

78. Silva JC. Levantamento e monitoramento da malacofauna de água doce da região de influência direta do Sistema Produtor do Alto Tietê (SPAT). [dissertação]. São Paulo: Universidade de São Paulo; 2010.

79. Silva RE, Melo AL, Pereira LH, Frederico LF. Levantamento malacológico da bacia hidrográfica do lago Soledade, Ouro Branco (Minas Gerais, Brasil). Rev Inst Med Trop Sao Paulo. 1994;36:437-44.

80. Silva-Santos IC, Laranja RK, Costa NC, Botelho GA, Ceresér VH, Martins JK. Lymnaea columella (Linnaeus, 1758) interagindo na disseminação da fasciolose na Estação Experimental Zootécnica de Dom Pedrito, RS. Hora Vet. 1987;35:42-8.

81. Silva-Santos IC, Albite-Silva FC, Pascotini LC. Lymnaea columella (Say, 1817) no município de Eldorado do Sul, Rio Grande do Sul. Hora Vet. 1994;14:30-2.

82. Simões RI. Comunidade de moluscos bentônicos na área de abrangência da usina hidrelétrica de Dona Francisca, Rio Jacuí, Rio Grande do Sul, Brasil: fase de pré e pós-enchimento do reservatório. [dissertação]. Porto Alegre: Universidade Federal do Rio Grande do Sul; 2002.

83. Sinclair KB. Pathogenesis of Fasciola and other liver - flukes. Heminthol Abstr. 1967;36:115-34.

84. Smooker PM, Hickford DE, Vaiano SA, Spithill TW. Isolation, cloning and expression of fatty acid binding proteins from Fasciola gigantica. Exp Parasitol. 1997;85:86-91.

85. Souza CP, Lima LC, Jannotti-Passos LK, Ferreira SS, Guimarães CT, Vieira IBF, et al. Moluscos límnicos da microrregião de Belo Horizonte, MG, com ênfase nos vetores de parasitoses. Rev Soc Bras Med Trop. 1998;31:449-56.

86. Souza CP, Magalhães KG, Jannotti-Passos LK, Santos GCP, Ribeiro F, Katz N. Aspects of the maintenance of the life cycle of Fasciola hepatica in Lymnaea columella in Minas Gerais, Brazil. Mem Inst Oswaldo Cruz. 2002;97:407-10.

87. Souza MAA, Souza LA, Machado-Coelho GLL, Melo AL. Levantamento malacológico e mapeamento das áreas de risco para transmissão da esquistossomose mansoni no município de Mariana, Minas Gerais, Brasil. Rev Ci Med Biol. 2006;5:132-9.

88. Teles HMS, Leite RPA, Rodrigues FL. Moluscos límnicos de uma área do Alto Araguaia (Brasil). Rev Saude Publica. 1991;25:179-83.

89. Thiengo SC, Fernandez MA, Boaventura MF, Stortti MA. A survey of freshwater gastropods in the microrregião serrana of the state of Rio de Janeiro, Brazil. Mem Inst Oswaldo Cruz. 1998;93(Suppl 1):233-4

90. Thiengo SC, Fernandez MA, Boaventura MF, Grault CE, Silva HFR, Mattos AC, et al. Freshwater snails and schistosomiasis mansoni in the state of Rio de Janeiro, Brazil. I - Metropolitan. Mem Inst Oswaldo Cruz. 2001;96(Suppl):177-84.

91. Thiengo SC, Fernandez MA, Boaventura MF, Santos SB, Mattos AC. Freshwater snails and schistosomiasis mansoni in the state of Rio de Janeiro, Brazil. II - Centro fluminense mesoregion. Mem Inst Oswaldo Cruz. 2002a;97:621-6.
92. Thiengo SC, Fernandez MA, Boaventura MF, Magalhães MG, Santos SB. Freshwater snails and schistosomiasis mansoni in the state of Rio de Janeiro, Brazil. III - Baixadas mesoregion. Mem Inst Oswaldo Cruz. 2002b;97( Suppl):43-6.

93. Thiengo SC, Mattos AC, Boaventura MF, Fernandez MA. Freshwater snails and Schistosomiasis mansoni in the state of Rio de Janeiro, Brazil. IV - Sul fluminense mesoregion. Mem Inst Oswaldo Cruz. 2004a;99:275-80.

94. Thiengo SC, Mattos AC, Boaventura MF, Loureiro MS, Santos SB, Fernandez MA Freshwater snails and schistosomiasis mansoni in the state of Rio de Janeiro, Brazil. V - Norte fluminense mesoregion. Mem Inst Oswaldo Cruz. 2004b;99(5 Suppl 1):99103.

95. Thiengo SC, Santos SB, Fernandez MA. Malacofauna límnica da área de influência do lago da usina hidrelétrica da Serra da Mesa, Goiás, Brasil. I. Estudo qualitativo. Rev Bras Zool. 2005;22:867-74

96. Thiengo SC, Mattos AC, Santos SB, Fernandez MA. Freshwater snails and schistosomiasis mansoni in the state of Rio de Janeiro, Brazil. VI - Noroeste fluminense mesoregion. Mem Inst Oswaldo Cruz. 2006;101(Suppl 1):239-45.

97. Tostes RA, Santarém VA, Alberti H, Sanches OC. Casos autóctones de Fasciola hepatica na região de Presidente Prudente, São Paulo, Brasil. Ci Rural. 2004;34:961-2.

98. Ueno H, Gutierres VC, Mattos MJT, Müller G. Fascioliasis problems in ruminants in Rio Grande do Sul, Brazil. Vet Parasitol. 1982;11:185-91.

99. Ueta MT. Alguns aspectos da biologia de Lymnaea columella Say, 1817 (Gastropoda, Pulmonata). Rev Saude Publica. 1976;10:355-66.

100. Ueta MT. Variações anatômicas em Lymnaea columella (Mollusca, Gastropoda). Rev Soc Bras Med Trop. 1977;11:217-37.

101. Ueta MT. Ocorrência de infecção natural de Fasciola hepatica Linnaeus, $1758 \mathrm{em}$ Lymnaea columella Say, 1817 no vale do Paraíba, SP, Brasil. Rev Saude Publica. $1980 ; 14: 230-3$

102. Vaz JF, Elmor MRD, Goncalves LMC, Ishihata GK. Resultados do levantamento planorbídico da área de Presidente Prudente, Estado de São Paulo. Rev Inst Med Trop Sao Paulo. 1983;25:120-6.

103. Vaz JF, Teles HM, Leite SP, Corrêa MA, Fabbro ALD, Rosa WS. Levantamento planorbídico do estado de São Paulo: sexta região administrativa. Rev Saude Publica. 1986;20:358-61.

104. Vaz JF, Mantegazza E, Teles HMS, Leite SPS, Morais LVC. Levantamento planorbídico do estado de São Paulo (Brasil): 4a região administrativa. Rev Saude Publica. 1987;21:371-9.

105. Vaz JF, Elmör MRD, Gonçalves LMC. Levantamento planorbídico do estado de São Paulo: 8a região administrativa (Grande área de São José do Rio Preto). Rev Inst Med Trop Sao Paulo. 1992;34:527-37.

106. Veitenheimer-Mendes IL, Lopes-Pitoni VL, da Silva MCP, Almeida-Caos JE, SchröderPfeiffer NT. Moluscos (Gastropoda e Bivalvia) ocorrentes nas nascentes do Rio Gravataí, Rio Grande do Sul, Brasil. Iheringia (Série Zool). 1992;73:69-76.

107. Vianna GJC, Lima LF, Paula EV, Melo AL. Levantamento preliminar dos gastrópodes límnicos no município de Januária, mesorregião norte de Minas Gerais, Brasil. In: XX Encontro Brasileiro de Malacologia. Rio de Janeiro; 2007. Livro de Resumos. p. 224. Available from: http://sbmalacologia.com.br/wp-content/uploads/2011/09/ Livro+de+Resumos+EBRAM+2007+Rio+de+Janeiro.pdf

108. World Health Organization. WHO. Triclabendazole and fascioliasis: a new drug to combat an age-old disease. Fact Sheets. 1998;191:1-4. Available from: http://www. who.ch/

Received: 19 December 2012

Accepted: 17 September 2013 